\title{
The residual finiteness of positive one-relator groups
}

\author{
Daniel T. Wise
}

\begin{abstract}
It is proven that every positive one-relator group which satisfies the $C^{\prime}\left(\frac{1}{6}\right)$ condition has a finite index subgroup which splits as a free product of two free groups amalgamating a finitely generated malnormal subgroup. As a consequence, it is shown that every $\mathrm{C}^{\prime}\left(\frac{1}{6}\right)$ positive one-relator group is residually finite. It is shown that positive one-relator groups are generically $\mathrm{C}^{\prime}\left(\frac{1}{6}\right)$ and hence generically residually finite. A new method is given for recognizing malnormal subgroups of free groups. This method employs a 'small cancellation theory' for maps between graphs.
\end{abstract}

Mathematics Subject Classification (2000). 20E26, 20F06, 20E06, $20 \mathrm{~F} 67$.

Keywords. Residually finite, one-relator group, malnormal, small cancellation theory.

\section{Introduction}

A one-relator presentation $\left\langle a, \ldots \mid \mathrm{W}^{n}\right\rangle$ is a positive one-relator presentation provided that $\mathrm{W}$ is a positive word, meaning that it is a word in the generators without any inverses, and $n \geq 1$. For example, badcab is a positive word, but $b a d c a^{-1} b$ is not. We will often use the term one-relator group for a group for which we have a specific one-relator presentation in mind. We now define the $\mathrm{C}^{\prime}(\alpha)$ small-cancellation condition for positive one-relator presentations. We refer the reader to [LS77] for a more general discussion of small-cancellation theory. A piece in a positive one-relator presentation $\left\langle a, \ldots \mid \mathrm{W}^{n}\right\rangle$ is a nontrivial word $\mathrm{P}$ which appears in two different ways as a subword of the cyclic word $\mathrm{W}$, where $\mathrm{W}$ is not a proper power. For instance, in the presentation $\langle a, b|$ abaaabbbaa $\rangle$, the pieces are $\{a, a a, a a a, b, b b, a b, b a, b a a, b a a a, b a a a b\}$. In the presentation $\langle a, b|$ $\left.a^{3} b^{2} a^{3} b^{2} a^{3} b^{2}\right\rangle$, the pieces are $\left\{a, b, a^{2}\right\}$.

A positive one-relator presentation $\left\langle a, \ldots \mid \mathrm{W}^{n}\right\rangle$ is said to satisfy the $\mathrm{C}^{\prime}(\alpha)$ small-cancellation condition provided that for each piece $\mathrm{P}$, we have $|\mathrm{P}|<\alpha\left|\mathrm{W}^{n}\right|$. For example, for each $m \geq 0$ let us examine the following positive one-relator group: $\mathrm{G}_{m}=\left\langle a, b \mid a^{1} b^{1} a^{2} b^{2} \ldots a^{m} b^{m}\right\rangle$. It is easy to verify that the longest piece in $\mathbf{G}_{m}$ is $a^{m-1} b^{m-1}$, and that consequently $\mathrm{G}_{m}$ satisfies the $\mathrm{C}^{\prime}\left(\frac{2 m-2}{m(m+1)-1}\right)$

\footnotetext{
Supported as an NSF Postdoctoral Fellow under grant no. DMS-9627506
} 
small-cancellation condition. In particular, $\mathrm{G}_{m}$ satisfies the $\mathrm{C}^{\prime}\left(\frac{1}{6}\right)$ condition for $m \geq 10$. Finitely presented groups which satisfy the $\mathrm{C}^{\prime}\left(\frac{1}{6}\right)$ condition are known to be word-hyperbolic (for instance, see Strebel's proof in the appendix to [GdlH90]).

Recall that a subgroup $\mathrm{M} \subset \mathrm{F}$ is malnormal if for each $f \in \mathrm{F}-\mathrm{M}$, the intersection of $\mathrm{M}$ and $f^{-1} \mathrm{M} f$ is trivial. The central result of this paper is:

Theorem 1.1. Let $\mathrm{G}$ be a positive one-relator group which satisfies the $\mathrm{C}^{\prime}\left(\frac{1}{6}\right)$ condition. Then $\mathrm{G}$ has a finite index subgroup which splits as a free product of two free groups amalgamating a finitely generated malnormal subgroup.

This splitting is obtained in Section 3 as part of Theorem 3.4, which also contains a splitting theorem under the weaker $\mathrm{C}^{\prime}\left(\frac{1}{4}\right)$ condition.

A group $\mathrm{G}$ is residually finite if for each nontrivial element $g \in \mathrm{G}$, there is a finite quotient $\mathrm{G} \rightarrow \overline{\mathrm{G}}$ such that $\bar{g}$ is nontrivial. Equivalently, $\mathrm{G}$ is residually finite if and only if the trivial subgroup is the intersection of finite index subgroups. We can now state the primary consequence of Theorem 1.1 which is:

Theorem 1.2. Let $\mathrm{G}$ be a positive one-relator group which satisfies the $\mathrm{C}^{\prime}\left(\frac{1}{6}\right)$ small-cancellation condition. Then $\mathrm{G}$ is residually finite.

A result of Baumslag's [Bau71] states that every positive one-relator groups is residually solvable. One might hope that Theorem 1.2 is deducible from Baumslag's theorem, or at least from its proof. However, Baumslag described an example due to Higman of a positive one-relator group which is not residually finite because it is not Hopfian. This example is reviewed below in Example 5.1.

In [Ego81], Egorov proved the residual finiteness of positive one-relator groups where the relator is of the form $\mathrm{W}^{n}$ and $n \geq 2$. Since a one-relator presentation $\left\langle a, \ldots \mid \mathrm{W}^{n}\right\rangle$ satisfies $\mathrm{C}^{\prime}\left(\frac{1}{n}\right)$, Egorov's result follows immediately from Theorem 1.2 for $n \geq 6$. For lower exponents, several additional arguments are required to obtain his result, but it essentially follows from the method of this paper and is completely reproved in Theorem 4.1.

In Section 3, we deduce Theorem 1.2 from Theorem 1.1 by applying the following theorem proven in [Wis]:

Theorem 1.3. Let $\mathrm{K}$ split as a free product of two free groups amalgamating a finitely generated malnormal subgroup, then $\mathrm{K}$ is residually finite.

In Section 2, we introduce the use of small-cancellation conditions to study maps $\phi: \mathrm{A} \rightarrow \mathrm{B}$ between graphs. We show that if $\phi: \mathrm{A} \rightarrow \mathrm{B}$ satisfies certain small-cancellation conditions then $\phi$ is $\pi_{1}$-injective, and $\phi_{*}\left(\pi_{1} \mathrm{~A}\right)$ is a malnormal subgroup of $\pi_{1} \mathrm{~B}$. This is employed in Section 3 to show that the amalgamated subgroup is malnormal.

In Section 4 we apply the method to one-relator groups with torsion.

In Section 5 we describe examples which indicate some of the limits for residual finiteness of positive one-relator groups, and some of the limits of our approach to this problem.

In Section 6 we show that in a suitable sense, positive one-relator groups are generically $\mathrm{C}^{\prime}\left(\frac{1}{6}\right)$ and hence, generically residually finite. 


\section{Small-cancellation conditions for graphs}

Definition 2.1. (Graph Terminology) A graph is a 1 -dimensional CW-complex. A map between graphs is combinatorial provided that 0 -cells are mapped to 0 cells, and open 1-cells are mapped homeomorphically to open 1 -cells. In this paper, each map $\phi: \mathrm{A} \rightarrow \mathrm{B}$ between graphs will have the property that, after we possibly subdivide A, the map $\phi$ is combinatorial. A map between graphs is an immersion if it is locally injective. Let $\mathrm{B} \rightarrow \mathrm{C}$ be a fixed map. For a map $\mathrm{A} \rightarrow \mathrm{B}$, its projection $\mathrm{A} \rightarrow \mathrm{C}$ is the composition $\mathrm{A} \rightarrow \mathrm{B} \rightarrow \mathrm{C}$.

An interval $\mathrm{P}$ is a compact connected real interval $[a, b] \subset \mathbb{R}$ with a graph structure. A path $\mathrm{P} \rightarrow \mathrm{B}$ is a map between graphs, where $\mathrm{P}$ is an interval. The path is trivial if $\mathrm{P}$ consists of a single vertex. The linear ordering of the real line gives every path an orientation, and we can therefore consider the initial and terminal vertices of $\mathrm{P}$ to be the least and greatest points in this ordering. We will generally use the terms initial vertex and terminal vertex to refer to the images of these points in $\mathrm{B}$. For a path $\mathrm{P} \rightarrow \mathrm{B}$ we write $\mathrm{P}^{-1}$ for the inverse of $\mathrm{P}$, which is the path with the opposite orientation. More precisely, $\mathrm{P}^{-1} \rightarrow \mathrm{B}$ is the composition $\mathrm{P} \rightarrow \mathrm{P} \rightarrow \mathrm{B}$ where $\mathrm{P} \rightarrow \mathrm{P}$ is the involution of $\mathrm{P}$ which reverses the orientation (if $\mathrm{P}$ is nontrivial). If $\mathrm{P} \rightarrow \mathrm{B}$ and $Q \rightarrow \mathrm{B}$ are paths and the terminal point of $\mathrm{P}$ is the same as the initial point of $Q$, then we write $\mathrm{P} Q$ for their concatenation. Each edge of a graph can be regarded as a path in an obvious way. By a circle we mean a graph homeomorphic to a topological circle, that is, some subdivision of the graph consisting of a single vertex and a single edge. We will often use the term immersed cycle for an immersed circle. We will use the notation $|\mathrm{P}|$ for the length of a path $\mathrm{P} \rightarrow \mathrm{A}$ which is the number $m$, such that $\mathrm{P}$ is the concatenation of $m$ edges of $\mathrm{A}$. We will occasionally use the notation $|\mathrm{P} \rightarrow \mathrm{A}|$ or $|\mathrm{P} \rightarrow \mathrm{B}|$ for the length when there are several paths with the same domain, and the meaning of $|\mathrm{P}|$ is not clear from the context. Let $X$ be the standard 2 -complex of the presentation $\left\langle a, \ldots \mid \mathrm{W}^{n}\right\rangle$. A word $U$ in $\left\{a^{ \pm 1}, \ldots\right\}$ determines a path in $X$, and conversely, combinatorial paths determine words. Similarly, cyclic words, and hence, a relator in the presentation determine closed paths. We will therefore use paths and words interchangeably in this case.

Definition 2.2. We say that $\mathrm{D} \subset \mathrm{A}^{(0)}$ is a set of distinguished vertices if each component of $\mathrm{A}-\mathrm{D}$ is homeomorphic to an open interval, and each end of this interval is connected to a vertex in $\mathrm{D}$. The connected components of $\mathrm{A}-\mathrm{D}$ determine paths $\mathrm{P} \rightarrow \mathrm{A}$ called arcs of $\mathrm{A}$. Observe that each arc embeds in $\mathrm{A}$ with the possible exception that its initial and terminal vertices might be the same. Note that for a graph $\mathrm{A}$, the set $\mathrm{D}=\mathrm{A}^{(0)}$ is a distinguished set of vertices for any subdivision $\mathrm{A}^{\prime}$ of $\mathrm{A}$. Unless otherwise indicated, the distinguished vertices of a graph will be the entire set of vertices, and its arcs will be its edges.

Definition 2.3. (Small-cancellation conditions for maps of graphs) Two paths $\mathrm{P}_{1} \rightarrow \mathrm{C}$ and $\mathrm{P}_{2} \rightarrow \mathrm{C}$ are equivalent if there exists an isomorphism $\mathrm{P}_{1} \rightarrow \mathrm{P}_{2}$ sending the initial point of $\mathrm{P}_{1}$ to the initial point of $\mathrm{P}_{2}$ such the diagram below 


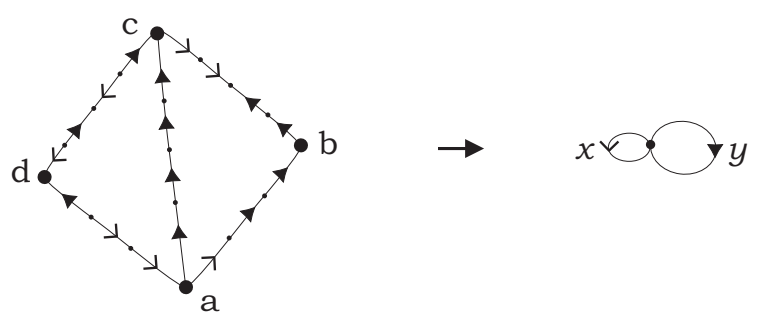

Figure 1. Consider the map $\mathrm{A} \rightarrow \mathrm{B}$ of graphs above. The large vertices of $\mathrm{A}$ labeled by $a$, $b, c$, and $d$ are distinguished. The two edges of $\mathrm{B}$ are labeled by $x$ and $y$. Consider the arc in A which join $a$ to $c$. It has two subpaths labeled yyy which form a piece-pair. While there are two paths in A labeled yyyy, they do not form a piece pair, because one of them passes through $b$ and is therefore not a subpath of an arc. The reader should verify that each arc in $\mathrm{A}$ is the concatenation of 2 pieces, and that the $c^{\prime}\left(\frac{4}{5}\right)$ condition is satisfied.

commutes. Otherwise, they are inequivalent.

$$
\begin{array}{cll}
\mathrm{P}_{1} & & \\
\downarrow & \searrow & \\
\mathrm{P}_{2} & \rightarrow & \mathrm{C}
\end{array}
$$

Let $\mathrm{A} \rightarrow \mathrm{B}$ be a map between graphs. Let $\mathrm{P}_{1} \rightarrow \mathrm{A}$ and $\mathrm{P}_{2} \rightarrow \mathrm{A}$ be subpaths of arcs of $\mathrm{A}$. Then $\mathrm{P}_{1} \rightarrow \mathrm{A}$ and $\mathrm{P}_{2} \rightarrow \mathrm{A}$ are a piece-pair if they are inequivalent but their projections $\mathrm{P}_{1} \rightarrow \mathrm{B}$ and $\mathrm{P}_{2} \rightarrow \mathrm{B}$ are equivalent. $\mathrm{A}$ path $\mathrm{P} \rightarrow \mathrm{A}$ is a piece if it is a member of a piece-pair.

We now define small cancellation conditions for a map $\mathrm{A} \rightarrow \mathrm{B}$ between graphs whose restriction to each arc of $\mathrm{A}$ is an immersion.

We say that $\mathrm{A} \rightarrow \mathrm{B}$ satisfies the $c(n)$ condition provided that no arc $\mathrm{E} \rightarrow \mathrm{A}$ is the concatenation of fewer than $n$ pieces.

The map $\mathrm{A} \rightarrow \mathrm{B}$ satisfies the $c^{\prime}(\alpha)$ condition provided that for each piece $\mathrm{P} \rightarrow \mathrm{A}$ which is a subpath of an arc $\mathrm{E} \rightarrow \mathrm{A}$, we have $|\mathrm{P} \rightarrow \mathrm{B}|<\alpha \mid \mathrm{E} \rightarrow$ $\mathrm{B} \mid$. Observe that if $\mathrm{A} \rightarrow \mathrm{B}$ satisfies $c^{\prime}\left(\frac{1}{n}\right)$ then $\mathrm{A} \rightarrow \mathrm{B}$ satisfies the $c(n+1)$ condition.

A cycle of $m$ pieces is a family of pieces $\mathrm{P}_{i} \rightarrow \mathrm{A}$ and $Q_{i} \rightarrow \mathrm{A}$ where $1 \leq i \leq$ $m$, such that the following conditions are satisfied for $1 \leq i \leq m$ : (coefficients are taken modulo $m$ ):

1. $\mathrm{P}_{i} \rightarrow \mathrm{A}$ and $Q_{i} \rightarrow \mathrm{A}$ are nontrivial subpaths of the same arc of $\mathrm{A}$.

2. $\mathrm{P}_{i} \rightarrow \mathrm{A}$ and $Q_{i} \rightarrow \mathrm{A}$ have the same terminal point, and the concatenation $\mathrm{P}_{i} Q_{i}^{-1} \rightarrow \mathrm{A}$ is an immersed path.

3. $\mathrm{P}_{i+1} \rightarrow \mathrm{A}$ and $Q_{i} \rightarrow \mathrm{A}$ form a piece-pair.

We say that $\mathrm{A} \rightarrow \mathrm{B}$ satisfies the $t(n)$ condition if there does not exist a cycle of $m$ pieces for any $m$ with $2<m<n$. We refer the reader to Figure 2 for a depiction of various cycles of pieces. The map $A_{5} \rightarrow T_{5}$ in Figure 6 satisfies $t(5)$ but not $t(4)$. To decide whether $\mathrm{A} \rightarrow \mathrm{B}$ satisfies the $t(n)$ condition, it is obviously sufficient to examine cycles of pieces where all the pieces $\mathrm{P}_{i}$ and $Q_{i}$ have length equal to one. 

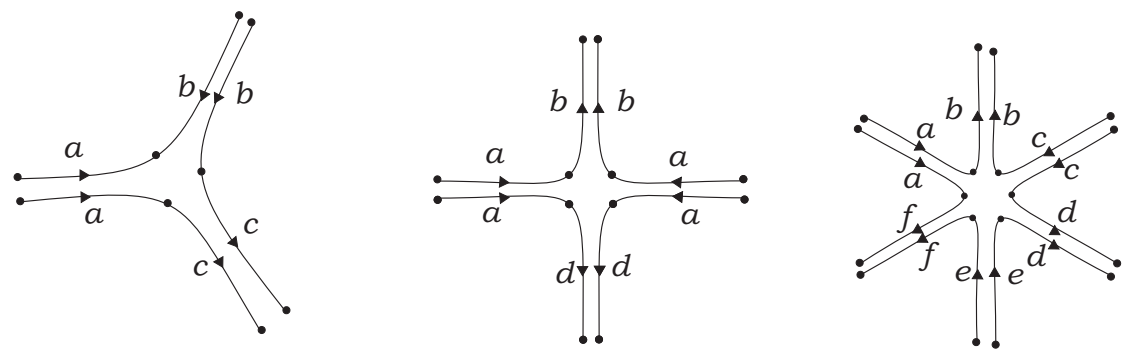

Figure 2. Depicted above are cycles of 3,4 , and 6 pieces.
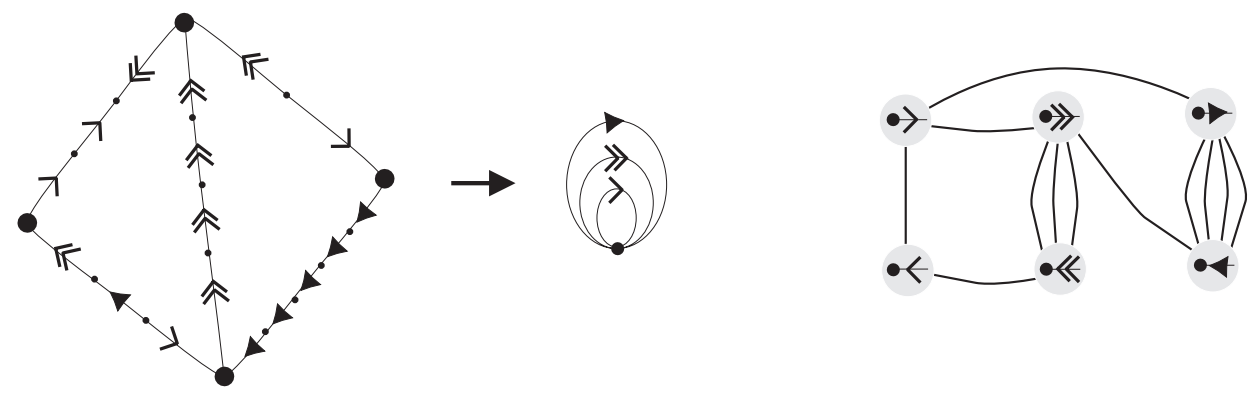

Figure 3. On the left is a map $\mathrm{A} \rightarrow \mathrm{B}$ between graphs. The distinguished vertices of $\mathrm{A}$ are the bold vertices. On the right is the cycle graph $\Lambda$ of this map. The map $\mathrm{A} \rightarrow \mathrm{B}$ satisfies the $t(4)$ condition because there is not immersed cycle in $\Lambda$ whose length is 3 .

An alternate formulation of the $t(n)$ condition goes as follows: We first ensure that $\mathrm{A}$ is subdivided so that the map $\phi: \mathrm{A} \rightarrow \mathrm{B}$ is combinatorial. We now define the cycle graph $\Lambda$ of the map $\phi: \mathrm{A} \rightarrow \mathrm{B}$ as follows: For each end $f$ of an edge of $\mathrm{B}$ there is a vertex $v_{f}$ in $\Lambda$. For each nondistinguished vertex $v$ of A there is an edge $e_{v}$ in $\Lambda$. If $g$ and $h$ are the ends of edges at $v$ in $\mathrm{A}$, then the edge $e_{v}$ is attached to the vertices $\phi(g)$ and $\phi(h)$ in $\Lambda$. (We use the notation $\phi(g)$ to denote the image in B of the end $g$.) We refer the reader to Figure 3 for an example of the cycle graph of a map between graphs. The map $\mathrm{A} \rightarrow \mathrm{B}$ satisfies the $t(n)$ condition if and only if the graph $\Lambda$ has no immersed cycles of length $m$ where $2<m<n$. We leave the equivalence of these two definitions of $t(n)$ to the reader.

Remark 2.4. One natural way to obtain maps between graphs is as follows: Any basis $\left\langle b_{1}, \ldots, b_{r}\right\rangle$ corresponds naturally to a bouquet of circles $\mathrm{B}$, which are directed and labeled by the $b_{i}$. Now, any word in $b_{i}^{ \pm 1}$ determines a closed path in $B$. Accordingly, given a set of words $\left\{W_{1}, \ldots, W_{n}\right\}$ in the generators, there is a bouquet $\mathrm{A}$ of $n$ circles corresponding to the $\mathrm{W}_{i}$, and a map $\mathrm{A} \rightarrow \mathrm{B}$ which sends the $i$-th loop of A to the closed path corresponding to $\mathrm{W}_{i}$. We say that $\left\{\mathrm{W}_{1}, \ldots, \mathrm{W}_{n}\right\}$ satisfies the $c(p)$ or $t(q)$ condition provided that the associated 
map $\mathrm{A} \rightarrow \mathrm{B}$ satisfies this condition.

The following two examples illustrate how the notion of piece for a map between graphs differs from the notion of piece in a presentation.

The set of words $\{a b a, a b b a\}$ determines a map from the bouquet of two circles to the bouquet of two circles. The pieces are $\{a, b, a b, b a\}$. If we use these words to form the presentation $\langle a, b \mid a b a, b a b\rangle$, then the pieces are $\{a, b, a b, b a, a a, b a a, a a b\}$.

The set $\left\{a^{10}\right\}$ consisting of a single word, determines a map from the bouquet of one circle to the one circle. The pieces of this map are paths corresponding to words $a^{m}$ where $m \leq 9$. In contrast, there are no pieces in the presentation $\left\langle a \mid a^{10}\right\rangle$.

Definition 2.5. (Orientation preserving) Let A and B be directed graphs. The map $\mathrm{A} \rightarrow \mathrm{B}$ is orientation preserving if each arc of $\mathrm{A}$ is mapped to the concatenation of consistently directed edges of $\mathrm{B}$.

Lemma 2.6. (Positive $\Rightarrow t(4))$ Let $\mathrm{A}$ and $\mathrm{B}$ be directed graphs. If $\mathrm{A} \rightarrow \mathrm{B}$ is orientation preserving then $\mathrm{A} \rightarrow \mathrm{B}$ satisfies the $t(4)$ condition. In particular, a set of positive words $\left\{\mathrm{W}_{i}\right\}$ in the generators $\left\{b_{j}\right\}$ satisfies the $t(4)$ condition.

Proof. Since A $\rightarrow$ B is orientation preserving, the ends at each nondistinguished vertex of A map to one incoming end in $\mathrm{B}$, and one outgoing end in $\mathrm{B}$. Consequently, the cycle graph of $\mathrm{A} \rightarrow \mathrm{B}$ is bipartite. Therefore the length of any immersed cycle in $\Lambda$ has even length, and we are done.

Definition 2.7. ( $\pi_{1}$-injective, Malnormal, Cyclonormal) Let $\phi: \mathrm{A} \rightarrow \mathrm{B}$ be a map of graphs. Note that we do not assume that A or B is connected. The map $\mathrm{A} \rightarrow \mathrm{B}$ is $\pi_{1}$-injective if each essential closed path in $\mathrm{A}$ is mapped to an essential closed path in B. An immersion $\phi: \mathrm{A} \rightarrow \mathrm{B}$ is malnormal if for any two distinct 0 -cells $a_{1}$ and $a_{2}$ which map to the same 0 -cell $b$ of $\mathrm{B}$, the intersection $\phi_{*} \pi_{1}\left(\mathrm{~A}, a_{1}\right) \cap \phi_{*} \pi_{1}\left(\mathrm{~A}, a_{2}\right)$ is trivial in $\pi_{1}(\mathrm{~B}, b)$. Similarly, the immersion $\phi: \mathrm{A} \rightarrow \mathrm{B}$ is cyclonormal if the intersection $\phi_{*} \pi_{1}\left(\mathrm{~A}, a_{1}\right) \cap \phi_{*} \pi_{1}\left(\mathrm{~A}, a_{2}\right)$ is either the trivial subgroup or a cyclic subgroup of $\pi_{1}(\mathrm{~B}, b)$. Note that in case $\mathrm{A}$ and $\mathrm{B}$ are connected, this definition agrees with the usual notion of $\pi_{1} \mathrm{~A}$ mapping to a malnormal (cyclonormal) subgroup of $\pi_{1} \mathrm{~B}$.

We briefly summarize Stallings' folding algorithm [Sta83] as follows:

Stallings' Folding Algorithm 2.8. The algorithm begins with a combinatorial map of graphs $\mathrm{A} \rightarrow \mathrm{B}$, and factors this map as a composition $\mathrm{A} \rightarrow \overline{\mathrm{A}} \rightarrow \mathrm{B}$ such that $\overline{\mathrm{A}} \rightarrow \mathrm{B}$ is an immersion of graphs and is thus $\pi_{1}$-injective, and such that $\mathrm{A} \rightarrow \overline{\mathrm{A}}$ is the composition $\mathrm{A}=\mathrm{A}_{1} \rightarrow \mathrm{A}_{2} \rightarrow \cdots \rightarrow \mathrm{A}_{s}=\overline{\mathrm{A}}$ where for $1 \leq i<s$, the map $\mathrm{A}_{i} \rightarrow \mathrm{A}_{i+1}$ is a folding map. The map $\mathrm{A}_{i} \rightarrow \mathrm{A}_{i+1}$ is a folding map if it is the obvious quotient obtained by identifying two edges which are incident at the same vertex of $\mathrm{A}_{i}$ and which map to the same edge of $\mathrm{B}$. Note that since each folding map is $\pi_{1}$-surjective, the map $\mathrm{A} \rightarrow \overline{\mathrm{A}}$ is $\pi_{1}$-surjective. Finally, note that when $\mathrm{A}$ and $\mathrm{B}$ are based and connected, the map $\mathrm{A} \rightarrow \overline{\mathrm{A}}$ is precisely the lift of $\mathrm{A}$ onto its image in $\hat{\mathrm{B}}$, where $\hat{\mathrm{B}}$ is the covering space corresponding to the image of $\pi_{1} \mathrm{~A}$ in $\pi_{1} \mathrm{~B}$. 


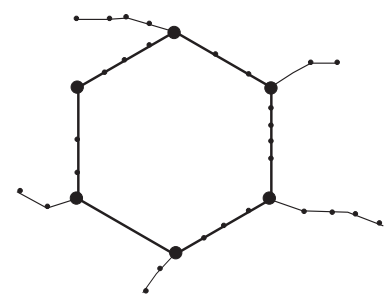

Figure 4. The six distinguished vertices in the circle of the crown above are the large bold vertices. The six segments are the arcs between them. Note that one of the tails is trivial.

Remark 2.9. If $\mathrm{A} \rightarrow \mathrm{B}$ is not $\pi_{1}$-injective, then there is an immersed circle $\mathrm{C} \rightarrow \mathrm{A}$ whose projection $\mathrm{C} \rightarrow \mathrm{B}$ is null-homotopic.

If $\mathrm{A} \rightarrow \mathrm{B}$ and hence $\overline{\mathrm{A}} \rightarrow \mathrm{B}$ is not malnormal then there are immersions of circles $\mathrm{C}_{1}^{\prime} \rightarrow \overline{\mathrm{A}}$ and $\mathrm{C}_{2}^{\prime} \rightarrow \overline{\mathrm{A}}$ which are inequivalent but whose projections $\mathrm{C}_{1}^{\prime} \rightarrow \mathrm{B}$ and $\mathrm{C}_{2}^{\prime} \rightarrow \mathrm{B}$ are equivalent. That is, there is no map $\mathrm{C}_{1}^{\prime} \rightarrow \mathrm{C}_{2}^{\prime}$ such that the diagram below on the left commutes, but there is a map $\mathrm{C}_{1}^{\prime} \rightarrow \mathrm{C}_{2}^{\prime}$ such that the diagram on the right commutes.

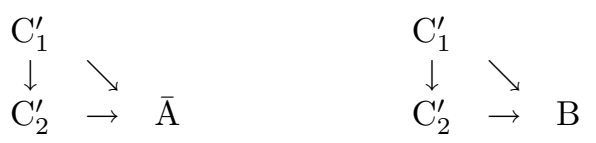

Definition 2.10. (crown) A crown $Q$ is a compact connected graph, such that $\chi(Q)=0$, and such that each vertex of $Q$ has valence $\leq 3$. The crown contains a circle $\mathrm{S}$ which contains some of the distinguished vertices of $Q$. The remaining distinguished vertices of $Q$ are the valence 1 vertices. For each distinguished vertex $v \in \mathrm{S}$, the component of $Q-(\mathrm{S}-v)$ containing $v$ is a tail of $Q$ beginning at $v$. A tail is trivial if it consists entirely of $v$. The arcs of $\mathrm{S}$ which join consecutive distinguished vertices of $\mathrm{S}$ are the segments of S. Figure 4 contains an illustration of a crown.

The following is a generalization of ideas of Nielsen (see [MKS66, Section 3.2]). The proof we present is more complicated than necessary so that it can serve as a warm-up towards the proof of Theorem 2.14.

Theorem 2.11 $\left(c(3) \Rightarrow \pi_{1}\right.$-injective). If $\mathrm{A} \rightarrow \mathrm{B}$ satisfies the $c(3)$ condition then $\mathrm{A} \rightarrow \mathrm{B}$ is $\pi_{1}$-injective.

Proof. The idea of the proof is to show that for any immersed circle $\mathrm{C} \rightarrow \mathrm{A}$, when we apply Stallings' algorithm to its projection $\mathrm{C} \rightarrow \mathrm{B}$ we obtain an immersed crown $\overline{\bar{C}} \rightarrow \mathrm{B}$. The map $\mathrm{C} \rightarrow \overline{\mathrm{C}}$ must therefore be $\pi_{1}$-injective, and so the composition $\mathrm{C} \rightarrow \overline{\mathrm{C}} \rightarrow \mathrm{B}$ is $\pi_{1}$-injective. The result follows by Remark 2.9.

Let $\mathrm{A} \rightarrow \mathrm{B}$ be a map between graphs which satisfies the $c(3)$ condition. Let $X, Y$, and $\mathrm{Z}$ be $\operatorname{arcs}$ of $\mathrm{A}$ with the property that the terminal point of $X$ is the initial point of $Y$ and the terminal point of $Y$ is the initial point of $\mathrm{Z}$, so that $X Y \mathrm{Z} \rightarrow \mathrm{A}$ is an immersed path. The $c(3)$ condition implies that $Y=U \mathrm{VW}$ 


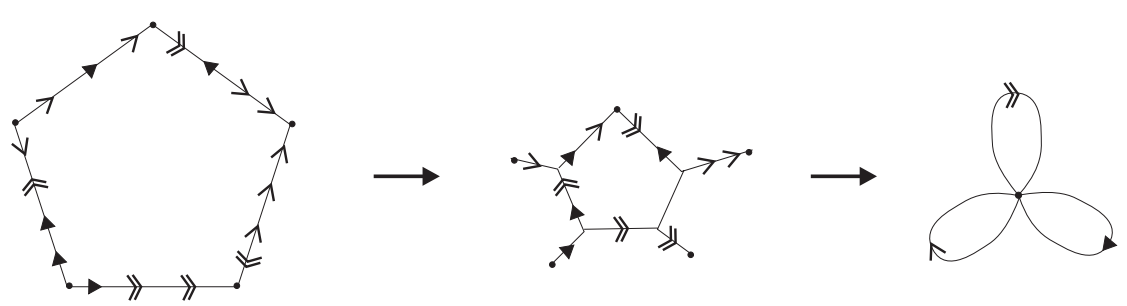

Figure 5. Illustrated above is $\mathrm{C} \rightarrow \overline{\mathrm{C}} \rightarrow \mathrm{B}$.

where $\mathrm{V}$ is a subpath of $Y$ with $|\mathrm{V}| \geq 1$, and $U$ is the maximal initial subpath of $Y$ which forms a piece-pair with an initial subpath of $X^{-1}$, and $\mathrm{W}$ is the maximal terminal subpath of $Y$ which forms a piece-pair with a terminal subpath of $\mathrm{Z}^{-1}$.

Now, the map $\mathrm{C} \rightarrow \mathrm{A}$ be an immersed circle which is the concatenation of arcs $Y_{1} \cdots Y_{n}$. As explained in the previous paragraph, the $c(3)$ condition implies that when we apply Stallings' folding algorithm to the projection $\mathrm{C} \rightarrow \mathrm{B}$, the initial subpath $U_{i}$ of $Y_{i}$ folds together with the terminal subpath $\mathrm{W}_{i-1}$ of $Y_{i-1}$, and the terminal subpaths $\mathrm{W}_{i}$ of $Y_{i}$ folds together with the initial subpath $U_{i+1}$ of $Y_{i+1}$. But the nontrivial intermediate arc $\mathrm{V}_{i}$ of $Y_{i}$ does not fold with anything.

Consequently, when Stallings' folding algorithm is applied to $\mathrm{C} \rightarrow \mathrm{B}$, we obtain an immersed crown $\overline{\mathrm{C}} \rightarrow \mathrm{B}$. The circle $\mathrm{C}^{\prime}$ of $\overline{\mathrm{C}}$ is the concatenation of segments $\mathrm{V}_{1} \cdots \mathrm{V}_{n}$ and the tails of $\overline{\mathrm{C}}$ correspond to the arcs $\mathrm{W}_{i}$ attached along the distinguished 0 -cells at the endpoints of these $i$ distinct segments. Note that the endpoint of $\mathrm{V}_{n}$ is attached to the initial point of $\mathrm{V}_{1}$, and we treat the coefficients modulo $n$. The composition $\mathrm{C} \rightarrow \overline{\mathrm{C}} \rightarrow \mathrm{B}$ is illustrated in Figure 5 .

Finally, $\mathrm{C} \rightarrow \overline{\mathrm{C}}$ obviously induces a $\pi_{1}$-isomorphism, and $\overline{\mathrm{C}} \rightarrow \mathrm{B}$ is an immersion and thus $\pi_{1}$-injective, so $\mathrm{C} \rightarrow \mathrm{B}$ is a $\pi_{1}$-injective and we are done.

Example 2.12. ([c(2)-t(n) $\nRightarrow \pi_{1}$-injective) For each $n$, let $\mathrm{T}_{n}$ denote the tree formed by wedging together $n$ edges, and let $\mathrm{A}_{n}$ denote the polygon with $n$ edges. As illustrated in Figure 6, there is a surjective map $\mathrm{A}_{n} \rightarrow \mathrm{T}_{n}$ which maps each edge of $\mathrm{A}_{n}$ to the concatenation of two edges of $\mathrm{T}_{n}$. For $n \geq 3$ the map $\mathrm{A}_{n} \rightarrow \mathrm{T}_{n}$ satisfies $c(2)-t(n)$, but obviously fails to be $\pi_{1}$-injective.

Our interest in the following lemma is a special case which asserts that given an immersed circle $\mathrm{C}^{\prime} \rightarrow \overline{\mathrm{A}}$, we can 'reverse the folding process', and obtain $\mathrm{C}^{\prime}$ as the circle of a crown $\overline{\mathrm{C}}$ which is itself obtained by applying Stallings' algorithm to the projection $\mathrm{C} \rightarrow \overline{\mathrm{A}}$ of an immersed circle $\mathrm{C} \rightarrow \mathrm{A}$.

Lemma 2.13. Let $\mathrm{A} \rightarrow \mathrm{B}$ satisfy the $c(3)$ condition, and let $\mathrm{A} \rightarrow \overline{\mathrm{A}} \rightarrow \mathrm{B}$ be the maps provided by Stallings' algorithm. Let $X^{\prime} \rightarrow \overline{\mathrm{A}}$ be an immersion, then there is an immersed graph $X \rightarrow \mathrm{A}$ such that we have the commutative diagram below, where $X^{\prime}$ embeds as a deformation retract of $\bar{X}$. Furthermore, if $X^{\prime} \rightarrow \overline{\mathrm{A}}$ is an immersed circle, then we can choose $X \rightarrow \mathrm{A}$ to be an immersed circle, and 

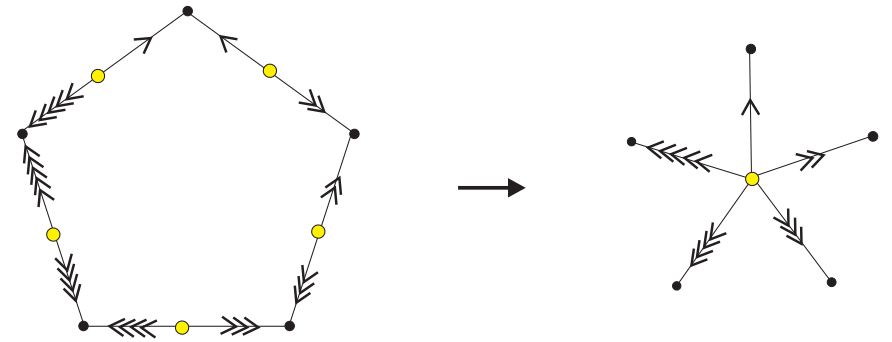

Figure 6. Illustrated above is the map $\mathrm{A}_{5} \rightarrow \mathrm{T}_{5}$.

$\bar{X}$ is a crown with circle $X^{\prime}$.

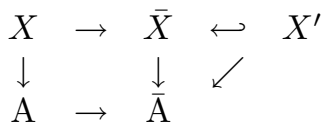

Proof. The proof is left to the reader.

Theorem 2.14. (Small cancellation $\Rightarrow$ malnormal) Suppose $\mathrm{A} \rightarrow \mathrm{B}$ satisfies $c(5)$ or $c(4)-t(4)$ or $c(3)-t(5)$. Then $\mathrm{A} \rightarrow \mathrm{B}$ is malnormal.

Proof. Suppose that $\overline{\mathrm{A}} \rightarrow \mathrm{B}$ is not malnormal, then by Remark 2.9, there are immersed circles $\mathrm{C}_{1}^{\prime} \rightarrow \overline{\mathrm{A}}$ and $\mathrm{C}_{2}^{\prime} \rightarrow \overline{\mathrm{A}}$ which are inequivalent, but whose projections to $\mathrm{B}$ are equivalent. By Lemma 2.13 for $i$ we have the following commutative diagram:

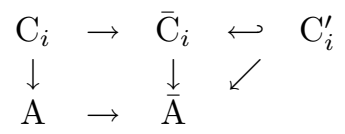

The arguments will depend on two observations. Firstly, as noted in the proof of Theorem 2.11, each tail of the crown $\overline{\mathrm{C}}_{i}$ corresponds to a piece-pair in the appropriate arcs of $\mathrm{C}_{i}$. Secondly, subsegments of the circles in $\overline{\mathrm{C}}_{1}$ and $\overline{\mathrm{C}}_{2}$ which correspond to each other under the isomorphism $\mathrm{C}_{1}^{\prime} \rightarrow \mathrm{C}_{2}^{\prime}$ determine pieces of the corresponding arcs in $\mathrm{C}_{1}$ and $\mathrm{C}_{2}$. Recall that segments were defined in Definition 2.10 to be the arcs joining consecutive distinguished vertices in the circle of a crown. By a subsegment we mean a subpath of a segment. The subsegments will arise by comparing corresponding circles in a pair of crowns (see Figure 7).

The c(5) case: Let $U$ be a segment of the crown $\overline{\mathrm{C}}_{1}$. Observe that the corresponding path in $\mathrm{C}_{2}^{\prime}$ is either the concatenation of successive subsegments $\mathrm{T}, \mathrm{V}$ of segments of $\overline{\mathrm{C}}_{2}$, or it contains an entire segment $\mathrm{W}$ of $\overline{\mathrm{C}}_{2}$. Consequently we see that either the arc of $\mathrm{C}_{1}$ corresponding to $U$ is the concatenation of at most 4 pieces, or the arc of $\mathrm{C}_{2}$ corresponding to $\mathrm{W}$ is the concatenation of at most 3 pieces. In either case, the $c(5)$ condition has been violated.

The $\mathbf{c}(4)-\mathbf{t}(4)$ case: First observe that each segment $U$ of $\overline{\mathrm{C}}_{1}$ has at least one distinguished point of $\overline{\mathrm{C}}_{2}$ in its interior. For otherwise, $U$ would be a subsegment of some segment of $\overline{\mathrm{C}}_{2}$ and so the arc of $\mathrm{C}_{1}$ corresponding to $U$ 


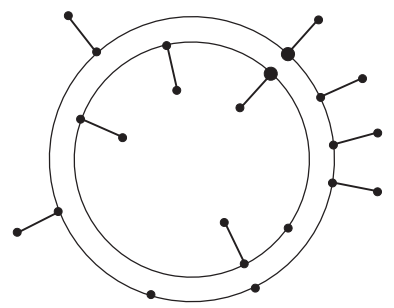

Figure 7. The outer crown is $\overline{\mathrm{C}}_{1}$ and the inner crown is $\overline{\mathrm{C}}_{2}$. We have drawn them so that the isomorphism between their circles $\mathrm{C}_{1}^{\prime}$ and $\mathrm{C}_{2}^{\prime}$ is the radial correspondence. All the bold vertices are distinguished. $\mathrm{C}_{1}$ consists of 8 arcs denoted by $\mathrm{E}_{1}, \ldots, \mathrm{E}_{8}$ beginning with the arc whose image contains the bold distinguished vertex, and proceeding in counterclockwise order. Similarly, we denote the arcs of $\mathrm{C}_{2}$ by $\mathrm{F}_{1}, \ldots, \mathrm{F}_{5}$. The isomorphism between $\mathrm{C}_{1}^{\prime}$ and $\mathrm{C}_{2}^{\prime}$ shows that $\mathrm{E}_{1}$ is the concatenation of 4 pieces. This is because the segment of $\mathrm{C}_{1}^{\prime}$ corresponding to $\mathrm{E}_{1}$ is the concatenation of 2 subsegments of $\mathrm{C}_{2}^{\prime}$, and the two tails in $\overline{\mathrm{C}}_{1}$ corresponding to $\mathrm{E}_{1}$, yield nontrivial pieces as well. Similarly $\mathrm{F}_{5}$ is the concatenation of 5 pieces because its corresponding segment in $\overline{\mathrm{C}}_{2}$ is the concatenation of 4 subsegments (three of which are entire segments) of $\mathrm{C}_{1}^{\prime}$, but only one of its tails is nontrivial. The reader should verify that the correspondence shows that $\mathrm{E}_{1}, \ldots, \mathrm{E}_{8}$ are the concatenations of 4,4 , $2,1,3,3,3$, and 3 pieces respectively. Similarly $\mathrm{F}_{1}, \ldots, \mathrm{F}_{5}$ are the concatenations of 3 , $4,5,2$, and 5 pieces respectively.

would be the concatenation of three pieces which contradicts the $c(4)$ hypothesis. The same statement holds with the roles of $\overline{\mathrm{C}}_{1}$ and $\overline{\mathrm{C}}_{2}$ reversed.

Next, observe that it follows from the $t(4)$ condition that if a distinguished point $p$ of $\overline{\mathrm{C}}_{1}$ is in the interior of a segment $U$ of $\overline{\mathrm{C}}_{2}$ then the tail in $\overline{\mathrm{C}}_{1}$ attached at $p$ is trivial. Again, the same statement holds with the roles of $\overline{\mathrm{C}}_{1}$ and $\overline{\mathrm{C}}_{2}$ reversed.

Combining the previous two observations we see that a segment $U$ of $\overline{\mathrm{C}}_{1}$ is the concatenation of two subsegments of $\overline{\mathrm{C}}_{2}$. If one of these is an entire segment of $\overline{\mathrm{C}}_{2}$, then the corresponding arc of $\mathrm{C}_{2}$ would be the concatenation of at most three pieces which is impossible. Consequently $U$ is the concatenation of proper subsegments of $\overline{\mathrm{C}}_{2}$, and so the second observation implies that the tails of the arc of $\mathrm{C}_{1}$ corresponding to $U$ are trivial. Therefore this arc is the concatenation of 2 pieces which is a contradiction.

The $\mathbf{c}(3)-\mathbf{t}(\mathbf{5})$ case: Observe that the $t(4)$ condition implies that if a distinguished point $p$ of $\overline{\mathrm{C}}_{1}$ lies in the interior of a segment of $\overline{\mathrm{C}}_{2}$ then the tail at $p$ is trivial. Similarly, if corresponding points $p_{1}$ and $p_{2}$ of $\mathrm{C}_{1}^{\prime}$ and $\mathrm{C}_{2}^{\prime}$ are distinguished, then the $t(5)$ condition insures that at most one of these points has a nontrivial tail. The same statement holds with the roles of $\overline{\mathrm{C}}_{1}$ and $\overline{\mathrm{C}}_{2}$ reversed.

No segment $U$ of $\overline{\mathrm{C}}_{1}$ contains more than one distinguished point of $\overline{\mathrm{C}}_{2}$ in its interior, for otherwise $U$ would contain an entire segment $\mathrm{V}$ of $\overline{\mathrm{C}}_{2}$ in its interior, and then since the tails at the endpoints of $\mathrm{V}$ must be trivial, we see that the arc of $\overline{\mathrm{C}}_{2}$ corresponding to $\mathrm{V}$ consists of a single piece, which violates the $c(2)$ condition.

No segment $U$ of $\overline{\mathrm{C}}_{1}$ contains exactly one distinguished point of $\overline{\mathrm{C}}_{2}$ in its 


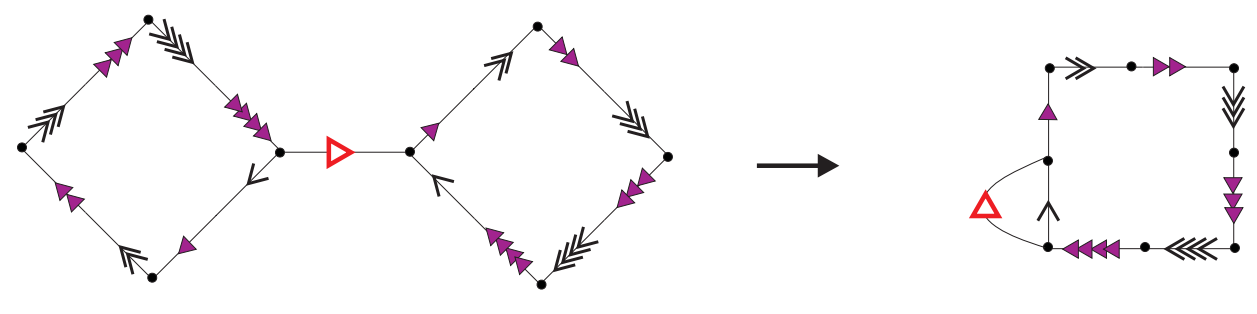

Figure 8. The map $\mathrm{A} \rightarrow \mathrm{B}$ above satisfies the $c(2)-t(4)$ condition, but it is not malnormal.

interior. Otherwise, either both endpoints of $U$ are contained in the interiors of segments of $\overline{\mathrm{C}}_{2}$ and thus have trivial tails, and so the arc of $\mathrm{C}_{1}$ corresponding to $U$ is the concatenation of 2 pieces which violates the $c(3)$ condition. Or, some segment $\mathrm{V}$ of $\overline{\mathrm{C}}_{2}$ is contained in $U$, with exactly one of its endpoints in the interior of $U$, in which case, the arc of $\mathrm{C}_{1}$ corresponding to $\mathrm{V}$, is the concatenation of at most 2 pieces which violates the $c(3)$ condition. Reversing the roles of $\mathrm{C}_{1}$ and $\mathrm{C}_{2}$, we see that no segment of $\overline{\mathrm{C}}_{2}$ can contain a distinguished point in its interior.

The only remaining possibility is that each segment $U$ of $\overline{\mathrm{C}}_{1}$ corresponds precisely to a segment $\mathrm{V}$ of $\overline{\mathrm{C}}_{2}$. But as shown above, the $c(5)$ condition implies that the endpoints of these segments contain at most one nontrivial tail in either $\overline{\mathrm{C}}_{1}$ or $\overline{\mathrm{C}}_{2}$ but not both. Since there are at most 2 tails, we see that either the arc corresponding to $U$ or the arc corresponding to $\mathrm{V}$ is the concatenation of at most 2 pieces. This violates the $c(3)$ condition, and we are done.

Remark 2.15. (Most subgroups are malnormal) In a 1998 lecture, G. Baumslag reported on an interesting discovery made using the Magnus group theory software. When the computer was instructed to select a large random sample of finitely generated subgroups of a rank 2 free group, the computer found that nearly all of these subgroups were malnormal. Perhaps Theorem 2.14 provides a satisfactory explanation for this phenomenon. Indeed, it is easy to believe that a typical finite set of words satisfies the $c(5)$ condition and hence generates a malnormal subgroup.

Example 2.16. ( $\pi_{1}$-injective \& $c(2)-t(n) \nRightarrow$ malnormal) We now give examples of maps $\mathrm{A} \rightarrow \mathrm{B}$ which are $\pi_{1}$-injective and satisfy $c(2)-t(n)$ but are not malnormal. Let $\mathrm{P}$ be a $2 n$-gon, and attach an edge $e$ from the first vertex to the second vertex of $\mathrm{P}$. Call the resulting graph $\mathrm{B}$. Let $\mathrm{A}$ denote two copies of $\mathrm{P}$ joined together by a single edge corresponding to $e$. There is an obvious immersion $\mathrm{A} \rightarrow \mathrm{B}$ which is illustrated in Figure 8 for the case $n=4$. Now, if we regard each copy of $\mathrm{P}$ as an $n$-gon each of whose sides is an arc of 2 -edges, then $\mathrm{A} \rightarrow \mathrm{B}$ satisfies $c(2)-t(n)$ and is $\pi_{1}$-injective but is not malnormal.

Example 2.17. ( $c(4) \nRightarrow$ malnormal) The following set of words satisfies the 


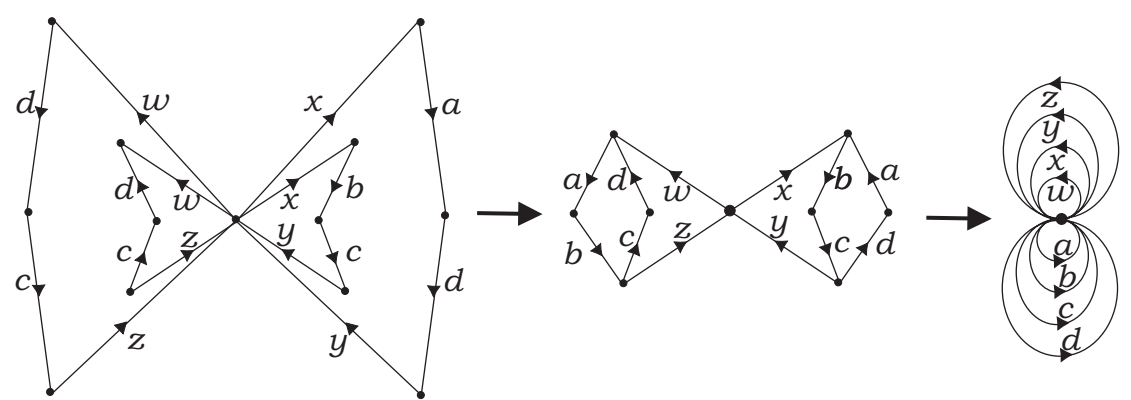

Figure 9. The above composition $\mathrm{A} \rightarrow \overline{\mathrm{A}} \rightarrow \mathrm{B}$ is obtained by applying Stallings' algorithm. A pair of abcd cycles in $\overline{\mathrm{A}}$ carries the $\mathbb{Z}$ subgroup in the intersection of conjugates.

$c(4)$ condition, but does not generate a malnormal subgroup.

$$
\left\{w a b z, w d^{-1} c^{-1} z, x b c y, x a^{-1} d^{-1} y\right\}
$$

Indeed, the conjugate of the element $(w a b z)\left(w d^{-1} c^{-1} z\right)^{-1}$ by $w a^{-1} x^{-1}$ is the element $(x b c y)\left(x a^{-1} d^{-1} y\right)^{-1}$. See Figure 9 for a depiction of the composition $\mathrm{A} \rightarrow \overline{\mathrm{A}} \rightarrow \mathrm{B}$ where $\mathrm{B}$ is a bouquet of circles labeled by the 8 generators, and $\mathrm{A} \rightarrow \mathrm{B}$ is the map of the the bouquet of four paths corresponding to the four generators.

Example 2.18. $(c(3)-t(4) \nRightarrow$ cyclonormal) The following set of words satisfies the $c(3)-t(4)$ condition, but does not generate a cyclonormal subgroup.

$$
\{x a y, x b y, x c z, x d z, r a s, r b s, r c t, r d t\}
$$

This is because if we conjugate the subgroup $\left\langle(x a y)(x b y)^{-1},(x c z)(x d z)^{-1}\right\rangle$ by the element $x r^{-1}$ then we obtain the subgroup $\left\langle(\right.$ ras $\left.)(r b s)^{-1},(r c t)(r d t)^{-1}\right\rangle$.

Proposition 2.19. ( $c(4) \Rightarrow$ cyclonormal) If $\mathrm{A} \rightarrow \mathrm{B}$ satisfies the $c(4)$ condition then $\mathrm{A} \rightarrow \mathrm{B}$ is cyclonormal.

Proof. (Sketch of proof) The proof is very similar to the proof of Theorem 2.14. If $\overline{\mathrm{A}} \rightarrow \mathrm{B}$ is not cyclonormal, then there are immersions $\mathrm{D}_{1}^{\prime} \rightarrow \overline{\mathrm{A}}$ and $\mathrm{D}_{2}^{\prime} \rightarrow \overline{\mathrm{A}}$ which are inequivalent, but whose projections $\mathrm{D}_{1}^{\prime} \rightarrow \mathrm{B}$ and $\mathrm{D}_{2}^{\prime} \rightarrow \mathrm{B}$ are equivalent. Furthermore $\mathrm{D}_{i}$ is compact, and $\chi\left(\mathrm{D}_{i}\right)=-1$. One then uses Lemma 2.13 to obtain the following diagram

$$
\begin{array}{ccccc}
\mathrm{D}_{i} & \rightarrow & \overline{\mathrm{D}}_{i} & \longleftarrow & \mathrm{D}_{i}^{\prime} \\
\downarrow & & \downarrow & \swarrow &
\end{array}
$$

Now one uses the correspondence between $\mathrm{D}_{1}^{\prime}$ and $\mathrm{D}_{2}^{\prime}$ to count pieces and show that the $c(4)$ conditions is violated. The details are left to the reader.

Remark 2.20. The results of this section provide an immediate way to glean algebraic information about the subgroup from geometric small cancellation information about the map. However, to put these results in proper perspective, it 
is important to note that for finite graphs, there is a simple way to determine if $\mathrm{A} \rightarrow \mathrm{B}$ is $\pi_{1}$-injective, malnormal or cyclonormal. We simply apply Stallings' folding algorithm [Sta83], to factor it as $\mathrm{A} \rightarrow \overline{\mathrm{A}} \rightarrow \mathrm{B}$.

The map $\mathrm{A} \rightarrow \mathrm{B}$ is $\pi_{1}$-injective if and only if $\mathrm{A} \rightarrow \overline{\mathrm{A}}$ is $\pi_{1}$-injective. This map fails to be $\pi_{1}$-injective if and only if at some step in the algorithm, the edges being folded together have exactly the same initial points and the same terminal points.

We then examine the non-diagonal components of the fiber product or pullback of $\overline{\mathrm{A}} \rightarrow \mathrm{B}$ with itself. The map $\overline{\mathrm{A}} \rightarrow \mathrm{B}$ is malnormal if and only if these components are trees. The map $\overline{\mathrm{A}} \rightarrow \mathrm{B}$ is cyclonormal if and only if each of these components has $\chi \geq 0$.

\section{Positive and $\mathrm{C}^{\prime}\left(\frac{1}{6}\right) \Rightarrow$ residually finite}

We begin the section with Construction 3.1 which gives a 'splitting' of a certain finite cyclic cover of the standard 2 -complex of a positive one-relator group. We then prove some lemmas which use the small cancellation theory developed in Section 2 to show that the attaching maps in the splitting are $\pi_{1}$-injective and malnormal. Finally we prove the residual finiteness theorem and several generalizations. The section is concluded by giving examples of one-relator presentations which satisfy small-cancellation conditions arbitrarily close to those in the hypothesis, but whose virtual splittings don't have the malnormality properties that we use to deduce the residual finiteness.

Construction 3.1. Let $X$ denote the standard 2 -complex of the one-relator presentation $\left\langle a, \ldots \mid \mathrm{W}^{n}\right\rangle$ where $\mathrm{W}^{n}$ is a positive word, $\mathrm{W}$ is not a proper power, and $n \geq 1$. Let $\hat{X} \rightarrow X$ denote the finite regular cover corresponding to the quotient $\pi_{1} X \rightarrow \mathbb{Z}_{\left|\mathrm{W}^{n}\right|}$ induced by the map which sends each generator of $\left\langle a, \ldots \mid \mathrm{W}^{n}\right\rangle$ to the generator 1 of $\mathbb{Z}_{\left|\mathrm{W}^{n}\right|}$.

The boundary of each 2 -cell of $\hat{X}$ determines a simple path in $\hat{X}^{(1)}$, and we say that two 2 -cells are equivalent provided that they have the same boundary. Note that there are exactly $n 2$-cells in each equivalence class, and each class corresponds to an orbit of a 2 -cell under the action of $\mathbb{Z}_{n} \subset \mathbb{Z}_{\mid \mathrm{W}^{n}}$ on $\hat{X}$. We identify all the 2 -cells in each equivalence class and we call the resulting complex $Y$. Note that $\pi_{1} Y=\pi_{1} \hat{X}$ because $Y$ is isomorphic to the subcomplex of $\hat{X}$ obtained by removing $n-1$ of the 2 -cells from each equivalence class.

The construction now depends upon whether or not $\left|\mathrm{W}^{n}\right|$ is even. We first consider the simpler case where $\left|\mathrm{W}^{n}\right|$ is even. Let $p$ and $q$ be a pair of antipodal vertices of $Y^{(1)}$. That is, $q=\frac{\left|\mathrm{W}^{n}\right|}{2} p$ where $\frac{\left|\mathrm{W}^{n}\right|}{2} \in \mathbb{Z}_{\left|\mathrm{W}^{n}\right|}$. If we cut $Y^{(1)}$ along $p$ and $q$ then we obtain a pair of graphs which we denote by $\mathrm{L}$ and $\mathrm{R}$, and let us assume that the names are chosen so that edges of $\mathrm{L}$ are directed from $p$ towards $q$, and edges of $\mathrm{R}$ are directed from $q$ towards $p$. See Figure 10 .

For each 2 -cell $k$ of $Y$ we choose a simple path $e_{k}$ intersecting $\partial k$ at $\{p, q\}$. 

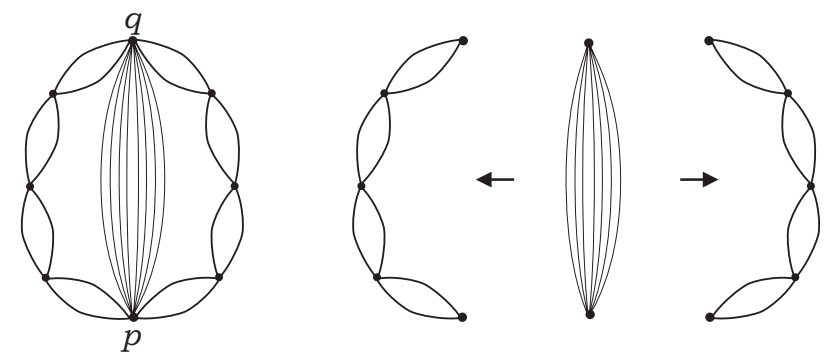

Figure 10. Illustrated above is the antipodal splitting coming from $\langle a, b \mid a b a b b a a b\rangle$ where the relator has an even length. Here $p$ and $q$ are antipodal, and $\mathrm{E}$ is the union of eight edges corresponding to the eight 2 -cells in $Y$. On the left is the graph $Y^{(1)} \cup \mathrm{E}$. On the right are the maps $\mathrm{L} \leftarrow \mathrm{E} \rightarrow \mathrm{R}$.

We let $\mathrm{E}$ denote the graph whose vertices are $\{p, q\}$ and whose edges are the paths $e_{k}$ where $k$ is a 2 -cell of $Y$. The reader may find it helpful to regard $\mathrm{E}$ as a graph in the subdivision of $Y$ which is induced by splitting each 2 -cell $k$ along an additional edge $e_{k}$. The resulting subdivision of $Y$ can then be split along the subgraph E.

We now consider the case where $\left|\mathrm{W}^{n}\right|$ is odd, and suppose that there are exactly $r$ generators in $\left\langle a, \ldots \mid \mathrm{W}^{n}\right\rangle$, or equivalently, $r$ edges in its standard 2 -complex $X$. In this case, we let $p$ be a vertex in $Y^{(1)}$ and we let $q_{1}, \ldots, q_{r}$ be points at the centers of the $r$ distinct edges of $Y^{(1)}$ which are antipodal to $p$. More precisely, for each $i$, the point $q_{i}$ lies at the center of an edge in $Y^{(1)}$ whose initial vertex is $\frac{\left|\mathrm{W}^{n}\right|-1}{2} p$. As in the even case, we cut $Y^{(1)}$ along $\left\{p, q_{1}, \ldots, q_{r}\right\}$ to obtain a pair of graphs which we denote by $\mathrm{L}$ and $\mathrm{R}$, and we assume that the names are chosen so that edges of $\mathrm{L}$ are directed from $p$ to the $q_{i}$, and edges of $\mathrm{R}$ are directed from the $q_{i}$ to $p$. See Figure 11. Finally, it will be convenient to subdivide each edge of $\mathrm{L}$ and $\mathrm{R}$ which is not incident with any $q_{i}$ vertex by adding a single vertex at its center. (This subdivision will simplify the statement and proof of Lemma 3.2.)

As in the even case, for each 2 -cell $k$ of $Y$ we choose a simple path $e_{k}$ intersecting $\partial k$ at $p$ and at exactly one of the points $\left\{q_{1}, \ldots, q_{r}\right\}$. We let $\mathrm{E}$ denote the graph whose vertices are $\left\{p, q_{1}, \ldots, q_{k}\right\}$ and whose edges are the paths $e_{k}$ where $k$ is a 2 -cell of $Y$.

We now continue the construction without distinguishing between the even and odd cases. Let $\mathrm{L}^{\prime}$ and $\mathrm{R}^{\prime}$ denote the closures of the components of $Y-\mathrm{E}$ containing $\mathrm{L}$ and $\mathrm{R}$. There are obvious deformation retractions $\mathrm{L}^{\prime} \rightarrow \mathrm{L}$ and $\mathrm{R}^{\prime} \rightarrow \mathrm{R}$ which are induced by pushing each edge $e_{k}$ of $\mathrm{E}$ to the left (or right) across the 2 -cell $k$. These induce maps $\mathrm{E} \rightarrow \mathrm{L}$ and $\mathrm{E} \rightarrow \mathrm{R}$ which are immersions on each arc of $\mathrm{E}$. Note that the restriction of $\mathrm{E} \rightarrow \mathrm{L}$ (respectively $\mathrm{E} \rightarrow \mathrm{R}$ ) to the arc $e_{k}$ yields a path which is essentially the left (right) half of the attaching map of the 2 -cell $k$. Choosing $p$ as the basepoint, van Kampen's theorem decomposes 

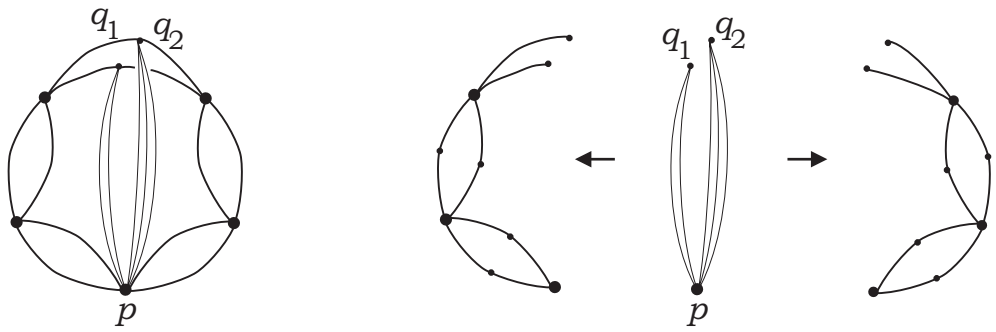

Figure 11. Illustrated above is the antipodal splitting coming from the presentation $\langle a, b \mid a b a b b\rangle$ whose relator has an odd length. Here $p$ is antipodal to $q_{1}$ and $q_{2}$, and $\mathrm{E}$ is the union of five edges corresponding to the five 2 -cells in $Y$. On the left is the graph $Y^{(1)} \cup \mathrm{E}$. On the right are the maps $\mathrm{L} \leftarrow \mathrm{E} \rightarrow \mathrm{R}$. Observe that the edges of $\mathrm{L}$ and $\mathrm{R}$ have been subdivided, and the new vertices are the small vertices.

$\pi_{1} Y$ as the following pushout:

$$
\begin{array}{ccc}
\pi_{1} \mathrm{E} & \rightarrow & \pi_{1} \mathrm{R} \\
\downarrow & & \downarrow \\
\pi_{1} \mathrm{~L} & \rightarrow & \pi_{1} Y
\end{array}
$$

Lemma 3.2. If $\left\langle a, \ldots \mid \mathrm{W}^{n}\right\rangle$ satisfies the $\mathrm{C}^{\prime}(\alpha)$ condition, then the maps $\mathrm{E} \rightarrow \mathrm{L}$ and $\mathrm{E} \rightarrow \mathrm{R}$ satisfy the $c^{\prime}(2 \alpha)$ condition.

Proof. We first consider the case where $\left|\mathrm{W}^{n}\right|$ is even. First note that for each arc $e_{f}$ of $\mathrm{E}$, the map $e_{f} \rightarrow \mathrm{L}$ is injective, and therefore there does not exist a piecepair consisting of subpaths of the same arc of $\mathrm{E}$. Now suppose that $\mathrm{P}_{f} \rightarrow \mathrm{E}$ and $\mathrm{P}_{g} \rightarrow \mathrm{E}$ form a piece-pair, and suppose that they are paths in the arcs $e_{f}$ and $e_{g}$ of $\mathrm{E}$, so $f$ and $g$ are distinct 2 -cells of $Y$. It follows that their projections, $\mathrm{P}_{f} \rightarrow X^{(1)}$ and $\mathrm{P}_{g} \rightarrow X^{(1)}$ yield a piece in the presentation $\left\langle a, \ldots \mid \mathrm{W}^{n}\right\rangle$, because they determine distinct occurrences of a word as a subword of the cyclic word $\mathrm{W}^{n}$. Indeed, the distinctness is guaranteed by our earlier observation that $f$ and $g$ are inequivalent cells. Consequently $\left|\mathrm{P}_{f}\right|<\alpha\left|\mathrm{W}^{n}\right|=2 \alpha\left|e_{f}\right|$ where $\left|e_{f}\right|$ is the length of the path $e_{f} \rightarrow \mathrm{L}$, and we are done. The same argument works for $\mathrm{E} \rightarrow \mathrm{R}$.

In case $\left|\mathrm{W}^{n}\right|$ is odd, a combinatorial path in $\mathrm{L}$ projects to a path in the standard 2 -complex which is the concatenation of half-edges. The length of a path $\mathrm{P} \rightarrow \mathrm{L}$ is exactly twice the length of its projection $\mathrm{P} \rightarrow X$, that is, $\mid \mathrm{P} \rightarrow$ $\mathrm{L}|=2| \mathrm{P} \rightarrow X \mid$. In particular, we note that $\left|e_{f}\right|=\left|\mathrm{W}^{n}\right|$. With this minor change and the obvious necessary definitional adjustments to allow the consideration of pieces which are the concatenation of half-edges, the same argument shows that $\left|\mathrm{P}_{f} \rightarrow \mathrm{L}\right|=2\left|\mathrm{P}_{f} \rightarrow X\right|<2 \alpha\left|\mathrm{W}^{n}\right|=2 \alpha\left|e_{f}\right|$ and we are done.

Lemma 3.3. If $\left\langle a, \ldots \mid \mathrm{W}^{n}\right\rangle$ is a positive one-relator group then $\mathrm{E} \rightarrow \mathrm{L}$ and $\mathrm{E} \rightarrow \mathrm{R}$ satisfy the $t(4)$ condition.

Proof. Since we directed the edges of $\mathrm{E}$ so that they originate at $p$ and terminate at $q$ (or $q_{i}$ in the odd case), the map $\mathrm{E} \rightarrow \mathrm{L}$ is orientation preserving. Similarly, 
if we reverse the direction on edges of $\mathrm{E}$, then the map $\mathrm{E} \rightarrow \mathrm{R}$ is orientation preserving. The result follows from Lemma 2.6.

Theorem 3.4. (Antipodal splitting) If $\left\langle a, \ldots \mid \mathrm{W}^{n}\right\rangle$ satisfies the $\mathrm{C}^{\prime}\left(\frac{1}{4}\right)$ condition, then $\pi_{1} \mathrm{E}$ injects in $\pi_{1} \mathrm{~L}$ and $\pi_{1} \mathrm{R}$, and we have a splitting of $\pi_{1} \hat{X}$ as an amalgamated free product of finitely generated free groups $\pi_{1} \mathrm{~L} *_{\pi_{1} \mathrm{E}} \pi_{1} \mathrm{R}$.

If $\left\langle a, \ldots \mid \mathrm{W}^{n}\right\rangle$ satisfies the $\mathrm{C}^{\prime}\left(\frac{1}{6}\right)$ condition, then $\pi_{1} \mathrm{E}$ is a malnormal subgroup of $\pi_{1} \mathrm{~L}$ and $\pi_{1} \mathrm{R}$.

Proof. By Lemma 3.2, $\mathrm{E} \rightarrow \mathrm{L}$ and $\mathrm{E} \rightarrow \mathrm{R}$ satisfy the $c^{\prime}\left(\frac{1}{2}\right)$ condition and thus satisfy the $c(3)$ condition. By Theorem 2.11, the maps $\pi_{1} \mathrm{E} \rightarrow \pi_{1} \mathrm{R}$ and $\pi_{1} \mathrm{E} \rightarrow$ $\pi_{1} \mathrm{~L}$ in Equation 3.1 are injective, and consequently $\pi_{1} \hat{X}$ is an amalgamated free product as claimed.

By Lemma 3.3, $\mathrm{E} \rightarrow \mathrm{L}$ and $\mathrm{E} \rightarrow \mathrm{R}$ satisfy the $t(4)$ condition. Now if $\left\langle a, \ldots \mid \mathrm{W}^{n}\right\rangle$ satisfies $\mathrm{C}^{\prime}\left(\frac{1}{6}\right)$, then by Lemma $3.2, \mathrm{E} \rightarrow \mathrm{L}$ and $\mathrm{E} \rightarrow \mathrm{R}$ satisfy $c^{\prime}\left(\frac{1}{3}\right)$. Consequently, they satisfy $c(4)-t(4)$ and so by Theorem $2.14, \mathrm{E} \rightarrow \mathrm{L}$ and $\mathrm{E} \rightarrow \mathrm{R}$ are malnormal.

Example 3.5. The positive one-relator group $\langle a, b \mid a a b b\rangle$ shows that Theorem 3.4 can fail under the slightest relaxation of the $C^{\prime}\left(\frac{1}{4}\right)$-hypothesis. Indeed, $\langle a, b \mid a a b b\rangle$ is commensurable with $\mathbb{Z} \times \mathbb{Z}$ and therefore does not contain a free group of rank 2 . In particular, it does not have an index 4 subgroup which splits over a free group of rank 3 as would be the case if the proof of Theorem 3.4 worked in this case. Note that the pieces of $\langle a, b \mid a a b b\rangle$ are all of length $\leq 1$ and therefore the presentation satisfies $\mathrm{C}^{\prime}\left(\frac{1}{4-\epsilon}\right)$ for any $\epsilon>0$.

Theorem 3.6. Suppose that the positive one-relator group $\left\langle a, \ldots \mid \mathrm{W}^{n}\right\rangle$ satisfies the $\mathrm{C}^{\prime}\left(\frac{1}{6}\right)$ small cancellation condition. Then it is residually finite.

Proof. Let $X$ denote the standard 2 -complex of $\left\langle a, \ldots \mid \mathrm{W}^{n}\right\rangle$ and let $\hat{X} \rightarrow$ $X$ be the finite covering space described in Construction 3.1. By Theorem 3.4, $\pi_{1} \hat{X}$ splits as the free product of free groups amalgamating a finitely generated malnormal subgroup. Theorem 1.3 asserts that $\pi_{1} \hat{X}$ is residually finite, Since $\pi_{1} X$ contains the finite index subgroup $\pi_{1} \hat{X}$ which is residually finite, we see that $\pi_{1} X$ itself must be residually finite.

The proof of Theorem 3.6 actually yields the following more general result:

Theorem 3.7. Let $\left\langle a, \ldots \mid \mathrm{W}_{1}^{n_{1}}, \ldots, \mathrm{W}_{k}^{n_{k}}\right\rangle$ be a finite presentation where the relators $\mathrm{W}_{i}^{n_{i}}$ are positive words of the same length. Suppose that the presentation satisfies $\mathrm{C}^{\prime}\left(\frac{1}{6}\right)$. Then the resulting group is residually finite.

The following gives another direction in which to generalize Theorem 3.6. Furthermore, the same argument proves a multi-relator version as in Theorem 3.7.

Theorem 3.8. Let $\left\langle a, \ldots \mid \mathrm{W}^{n}\right\rangle$ be a positive one-relator group. Suppose that for each subword $Q$ of $\mathrm{W}^{n}$, if $|Q|>\frac{1}{2}\left|\mathrm{~W}^{n}\right|$ then $Q$ is not the concatenation of fewer than 4 pieces of $\mathrm{W}^{n}$. Then the group is residually finite. 
We note that the condition in Theorem 3.8 holds if each piece $\mathrm{P}$ in $\mathrm{W}^{n}$ satisfies $|\mathrm{P}| \leq \frac{1}{6}\left|\mathrm{~W}^{n}\right|$.

Proof. Without loss of generality, we may assume that $\left|\mathrm{W}^{n}\right|$ is even. To see this, observe that we can form a 2 -complex $X^{\prime}$ by identifying the center of each edge of $X$ with the 0 -cell of $X$, and $\pi_{1} X^{\prime} \cong \pi_{1} X * \mathrm{~F}$ where $\mathrm{F}$ is a free group. Since a subgroup of a residually finite group is itself residually finite, we see that it is sufficient to show that $\pi_{1} X^{\prime}$ is residually finite. Furthermore, $X^{\prime}$ is the standard 2 -complex of a one-relator group $\left\langle a^{\prime}, \ldots,\left(\mathrm{W}^{\prime}\right)^{n}\right.$ whose relator has even length.

The strategy of the proof is the same as for Theorem 3.6. The main difference is that we will apply a variant of Construction 3.1. Let $\hat{X}$ denote the $\mathbb{Z}_{\left|\mathrm{W}^{n}\right|}$ cover, and let $Y \subset \hat{X}$ be the subcomplex obtained by removing various 2 -cells as in Construction 3.1.

We form a new 2 -complex $Y^{\prime}$ which is a quotient of $Y$ obtained by identifying the centers of certain edges. Since $\pi_{1} Y^{\prime}=\pi_{1} Y * \mathrm{~F}$ where $\mathrm{F}$ is a free group, it will be sufficient to prove that $\pi_{1} Y^{\prime}$ is residually finite. Consider the set of points at centers of edges of $Y$. Let us say that two such points are equivalent if their corresponding edges have the same initial vertex (and hence the same terminal vertex). We then obtain $Y^{\prime}$ from $Y$ by identifying all the points in each equivalence class. We will refer to each such resulting point as an identification vertex of $Y^{\prime}$, and we note that $Y^{\prime}$ is a combinatorial 2 -complex once we declare these identification vertices to be vertices.

Let $p$ and $q$ be antipodal identification vertices in $Y^{\prime}$. We then obtain graphs $\mathrm{L}, \mathrm{R}$, and $\mathrm{E}$, and maps $\mathrm{E} \rightarrow \mathrm{L}$ and $\mathrm{E} \rightarrow \mathrm{R}$ as in Construction 3.1. The proof will be the same as for Theorem 3.6 once we justify that $\mathrm{E} \rightarrow \mathrm{L}$ (and similarly $\mathrm{E} \rightarrow \mathrm{R}$ ) satisfies the $c(4)$ condition:

Suppose that $\mathrm{E} \rightarrow \mathrm{L}$ does not satisfy the $c(4)$ condition. Then there is an arc $\mathrm{A}$ of $\mathrm{E}$ such that $\mathrm{A} \rightarrow \mathrm{L}$ is the concatenation $\mathrm{P}_{1} \mathrm{P}_{2} \mathrm{P}_{3}$ where each $\mathrm{P}_{i}$ is a piece. The fundamental observation is that since $\mathrm{P}_{1}$ starts at the identification vertex $p$, there exists an edge $e$ in $Y^{\prime}$ such that the concatenation $\mathrm{P}_{1}^{\prime}=e \mathrm{P}_{1}$ projects to a presentation-piece. Similarly, there is an edge $f$ in $Y^{\prime}$ such that the concatenation $\mathrm{P}_{3}^{\prime}=\mathrm{P}_{3} f$ projects to a presentation-piece. Let $Q$ be the concatenation $e \mathrm{~A} f$ and observe that $|Q|=|e \mathrm{~A} f|=1+\frac{\left|\mathrm{W}^{n}\right|}{2}+1>\frac{\left|\mathrm{W}^{n}\right|}{2}$. Finally, observe that $Q$ is the concatenation $\mathrm{P}_{1}^{\prime} \mathrm{P}_{2} \mathrm{P}_{3}^{\prime}$ which is a contradiction.

Remark 3.9. The reader can verify that the construction used in the proof of Theorem 3.8 shows that a positive one-relator group which satisfies the $\mathrm{C}^{\prime}\left(\frac{1}{4}\right)$ condition has a finite index subgroup which splits as a graph of free groups. Indeed, this is often true without any small-cancellation condition. In particular, for the example $\langle a, b \mid a a b b\rangle$ of Example 3.5, the construction induces a splitting whose underlying graph is a circle containing two edges, and whose edge and vertex groups are cyclic.

Remark 3.10. Let $X$ be the standard 2 -complex of a positive one-relator group 
$\left\langle a, \ldots \mid \mathrm{W}^{n}\right\rangle$. Let $\hat{X}, Y, \mathrm{E} \rightarrow \mathrm{L}$ and $\mathrm{E} \rightarrow \mathrm{R}$ be as in Construction 3.1. Suppose that $\mathrm{E} \rightarrow \mathrm{L}$ and $\mathrm{E} \rightarrow \mathrm{R}$ are $\pi_{1}$-injective. Because these maps are not arbitrary maps, it is reasonable to ask whether they must be malnormal as well. As illustrated by the examples below, it turns out that for each $\epsilon>0$, the $\mathrm{C}^{\prime}\left(\frac{1}{6-\epsilon}\right)$ condition is insufficient to provide malnormality.

Despite these examples, it seems that even among the class of positive onerelator groups that don't even satisfy $\mathrm{C}^{\prime}\left(\frac{1}{4}\right)$, the construction generically produces maps $\mathrm{E} \rightarrow \mathrm{L}$ and $\mathrm{E} \rightarrow \mathrm{R}$ which are $\pi_{1}$-injective and malnormal, and hence $\pi_{1} X$ is residually finite.

Suppose that the finite presentation $\left\langle a, \ldots \mid \mathrm{W}_{1}, \ldots, \mathrm{W}_{k}\right\rangle$ satisfies the $\mathrm{C}^{\prime}\left(\frac{1}{4}\right)$ condition, and the words $\mathrm{W}_{i}$ are positive and of the same length. A simple way to recognize that the amalgamated subgroup given by Theorem 3.4 is not malnormal is to find words $\mathrm{A}, \mathrm{B}, \mathrm{S}, \mathrm{T}, U, \mathrm{~V}$ such that

1. $|\mathrm{A}|=|\mathrm{B}|,|\mathrm{S}|=|\mathrm{T}|,|U|=|\mathrm{V}|$ and

2. $|\mathrm{A}|+|\mathrm{S}|+|U|=\frac{1}{2}\left|\mathrm{~W}_{i}\right|$ and

3. $\mathrm{SA} U, \mathrm{SB} U, \mathrm{TAV}, \mathrm{TBV}$ occur in distinct ways as subwords of the relators.

If there are such subwords, then after applying Stallings' algorithm to the map $\mathrm{E} \rightarrow \mathrm{L}$, we see that $\overline{\mathrm{E}}$ has two distinct cycles of length 2 which both project to a cycle with label $\mathrm{AB}^{-1}$ in $\mathrm{L}$. A similar argument shows that $\mathrm{E} \rightarrow \mathrm{R}$ is not malnormal.

I do not know if there is a positive one-relator group whose largest piece is exactly $\frac{1}{6}$ the length of the relator, but such that the antipodal splitting of Theorem 3.6 does not have a malnormal amalgamated subgroup. The following is an example of a positive two-relator group where the splitting does not have a malnormal amalgamated subgroup. I do not know if there is such an example in the combinatorially more restrictive one-relator case. Nevertheless, as shown in the proof of Theorem 3.8, there is a variant of the antipodal splitting with the necessary properties, and so we obtain the desired residual finiteness conclusion in the $\leq \frac{1}{6}$ case.

Example 3.11. Consider the following presentation:

$$
\mathrm{P}=\langle a, b, x, y, z| \text { axayaz, bxbybz }\rangle
$$

Each piece has length at most one sixth the length of a relator. The presentation therefore satisfies $\mathrm{C}^{\prime}\left(\frac{1}{6-\epsilon}\right)$ for each $\epsilon>0$. Though the proof of Theorem 3.4 provides a splitting, the amalgamated subgroup is not even cyclonormal because its intersection with one of its conjugates contains a free group of rank 2 . This is indicated by the subwords axa, aya, $a z a$ and $b x b, b y b, b z b$ of the relators.

We now give an infinite family of one-relator presentations which show that for each $\epsilon>0$ the $\mathrm{C}^{\prime}\left(\frac{1}{6-\epsilon}\right)$ condition is inadequate to prove that the amalgamated subgroup in the antipodal splitting is malnormal.

Example 3.12. For $m \geq 1$, let $\mathrm{P}_{m}$ denote the following presentation:

$$
\left\langle a, b, c \mid(a b)^{m} a^{2 m+1}(b a)^{m} b^{2 m+1}(a b)^{m} c^{2 m-2}\right\rangle
$$


Observe that any piece in $\mathrm{P}_{m}$ is a proper subword of $a^{2 m+1}, b^{2 m+1}, c^{2 m-2}$, or $(a b)^{m+1}$. Therefore, since the relator has length $12 m$, we see that $\mathrm{P}_{m}$ satisfies $\mathrm{C}^{\prime}\left(\frac{2 m+2}{12 m}\right)=\mathrm{C}^{\prime}\left(\frac{m+1}{6 m}\right)$. However, $\mathrm{P}_{m}$ satisfies the condition given above for failure of malnormality of the amalgamated subgroup in the splitting. Indeed, the subwords ALB, AMB, ULV, UMV are indicated below in brackets:

$$
\begin{aligned}
& {\left[(a b)^{m}\right]\left[a^{2 m}\right]\left[(a b)^{m}\right] a b^{2 m+1}(a b)^{m} c^{2 m-2}} \\
& (a b)^{m} a^{2 m+1} b\left[(a b)^{m}\right]\left[b^{2 m}\right]\left[(a b)^{m}\right] c^{2 m-2} \\
& a\left[(b a)^{m}\right]\left[a^{2 m}\right]\left[(b a)^{m}\right] b^{2 m+1}(a b)^{m} c^{2 m-2} \\
& (a b)^{m} a^{2 m+1}\left[(b a)^{m}\right]\left[b^{2 m}\right]\left[(b a)^{m}\right] b c^{2 m-2}
\end{aligned}
$$

While the examples above indicate that an analysis of the $\mathrm{C}^{\prime}\left(\frac{1}{4}\right)$ case will be more complex than the $\mathrm{C}^{\prime}\left(\frac{1}{6}\right)$ case, I do expect a positive resolution to the following:

Conjecture 3.13. Let $\mathrm{G}=\left\langle a, \ldots \mid \mathrm{W}^{n}\right\rangle$ be a positive one-relator group. If $\mathrm{G}$ satisfies the $\mathrm{C}^{\prime}\left(\frac{1}{4}\right)$ small-cancellation condition, then $\mathrm{G}$ is residually finite.

\section{Positive one-relator groups with torsion}

In this section we apply the method of Section 3 to the special case of positive one-relator groups with torsion. As mentioned in the introduction, these were shown to be residually finite in [Ego81]. We remark that a well-known conjecture of G. Baumslag's asserts that every one-relator group with torsion is residually finite [Bau67].

Theorem 4.1. Let $\mathrm{G}=\left\langle a, \ldots \mid \mathrm{W}^{n}\right\rangle$ where $\mathrm{W}$ is a positive word. If $n \geq 2$ then $\mathrm{G}$ is residually finite.

Proof. The proof is broken into several cases depending upon $n$.

$\mathbf{n} \geq \mathbf{6}$ : We first consider the case $n \geq 6$, since it follows directly from the statement of Theorem 3.6. Indeed, any piece $\mathrm{P}$ in the presentation $\left\langle a, \ldots \mid \mathrm{W}^{n}\right\rangle$ has the property that $|\mathrm{P}|<|\mathrm{W}|$ and consequently, the presentation satisfies the $\mathrm{C}^{\prime}\left(\frac{1}{n}\right)$ condition.

$\mathbf{n} \geq 4$ : We now prove the result for $n \geq 4$ by following the proof of Theorem 3.6, but replacing the $\mathrm{C}^{\prime}\left(\frac{1}{6}\right)$ condition by a result of Pride's [Pri83] which states that the one-relator group $\left\langle a, \ldots \mid \mathrm{W}^{n}\right\rangle$ satisfies the $\mathrm{C}(2 n)$ small-cancellation condition. It follows that any subword of $\mathrm{W}^{n}$ which is of length $\geq 2|\mathrm{~W}|$ is not the concatenation of fewer than 4 pieces. Thus, for $n \geq 4$, the maps $\mathrm{E} \rightarrow \mathrm{L}$ and $\mathrm{E} \rightarrow \mathrm{R}$ satisfy the $c(4)$ condition. Specifically, if some arc was the concatenation of at most three pieces, then some cyclic permutation of $\mathrm{W}^{2}$ would be the concatenation of at most three pieces. But this contradicts Pride's result that $\left\langle a, \ldots \mid \mathrm{W}^{2}\right\rangle$ satisfies $\mathrm{C}(4)$. The remainder of the proof for $n \geq 4$ is the same as the proof of Theorem 3.6. 


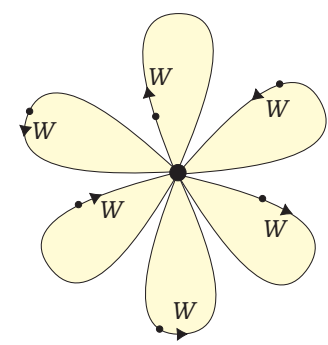

Figure 12. Illustrated above is the disc diagram consisting of a cyclically ordered arrangement of six 2 -cells around the a base 0 -cell. The path around each 2 -cell which starts at the small bold vertex and travels in the direction of the arrow, corresponds to the word $\mathrm{W}$. The fact that no clockwise path around a 2 -cell is inverse to the clockwise path around the next 2 -cell, is indicated by the disagreement between the positions of small bold vertices in consecutive 2 -cells.

$\mathbf{n}=\mathbf{2}$ : We now prove the theorem in the case $n=2$, and we assume that $\mathrm{W}$ is not a proper power. Let $X$ be the standard 2 -complex of $\left\langle a, \ldots \mid \mathrm{W}^{2}\right\rangle$, and let $\hat{X}, Y, \mathrm{E}, \mathrm{L}$ and $\mathrm{R}$ be as in Construction 3.1. Let $\mathrm{Z}$ be the standard 2 -complex of $\langle a, \ldots \mid \mathrm{W}\rangle$, and let $\hat{\mathrm{Z}}$ be the cover of $\mathrm{Z}$ corresponding to the obvious $\mathbb{Z}_{|\mathrm{W}|}$ cover of $\mathrm{Z}$. Observe that there is a natural immersion $\mathrm{L} \rightarrow \hat{\mathrm{Z}}$ and hence a map $\mathrm{E} \rightarrow \hat{\mathrm{Z}}$.

We will show that $\mathrm{E} \rightarrow \mathrm{L}$ (or similarly $\mathrm{E} \rightarrow \mathrm{R}$ ) is $\pi_{1}$-injective and malnormal, and so $\pi_{1} X$ is residually finite. The proof will not employ the small cancellation criterion. Instead, we will make fundamental use of the fact that $\mathrm{Z}$ and hence $\hat{\mathrm{Z}}$ is diagrammatically reducible, which means that any combinatorial map $\mathrm{S}^{2} \rightarrow \hat{\mathrm{Z}}$ has a cancelable pair of 2 -cells. We refer the reader to [BP93] for the basic notions regarding diagrammatic reducibility.

Observe that $\mathrm{E} \rightarrow \mathrm{L}$ is $\pi_{1}$-injective, if and only if the projection $\mathrm{E} \rightarrow \hat{\mathrm{Z}}$ is $\pi_{1}$-injective. Suppose that there is an immersed closed path $\mathrm{P} \rightarrow \mathrm{E}$ whose projection $\mathrm{P} \rightarrow \hat{\mathrm{Z}}$ is nullhomotopic, then there is an immersed circle $\mathrm{C} \rightarrow \mathrm{E}$ whose projection $\mathrm{C} \rightarrow \hat{\mathrm{Z}}$ is nullhomotopic and such that $|\mathrm{C}| \leq|\mathrm{P}|$. Let us assume that the length $|\mathrm{C}|$ of $\mathrm{C}$ is minimal among all such immersed circles. Each arc of $\mathrm{E}$ corresponds to a distinct cyclic permutation of the word $\mathrm{W}$, and since $\mathrm{C} \rightarrow \mathrm{E}$ is an immersion, no two consecutive arcs of $\mathrm{C}$ project to paths in $\hat{Z}$ which are inverse to each other. This sequence of cyclic permutations of $\mathrm{W}$ corresponds to a based disc diagram $\mathrm{D}$ in $\hat{\mathrm{Z}}$ which consists of a cyclically ordered arrangement of 2 -cells arranged around a base 0 -cell, as in Figure 12, such that the (clockwise) boundary paths of consecutive 2 -cells beginning at the basepoint are not inverse to each other. Since $\mathrm{C} \rightarrow \hat{\mathrm{Z}}$ is nullhomotopic, it is freely equivalent in $\hat{\mathrm{Z}}$ to the identity, and since $\mathrm{C}$ is the boundary path of $\mathrm{D}$, after successively folding pairs of edges we obtain a sphere $\mathrm{S}^{2}$ and a map $\mathrm{S}^{2} \rightarrow \hat{\mathrm{Z}}$.

We will now use the diagrammatic reducibility to complete the proof. First note that there does not exist an essential length 2 circle $\mathrm{C} \rightarrow \mathrm{E}$ whose projection $\mathrm{C} \rightarrow \hat{\mathrm{Z}}$ is nullhomotopic. Indeed, in this case, the cancelable pair in the resulting 

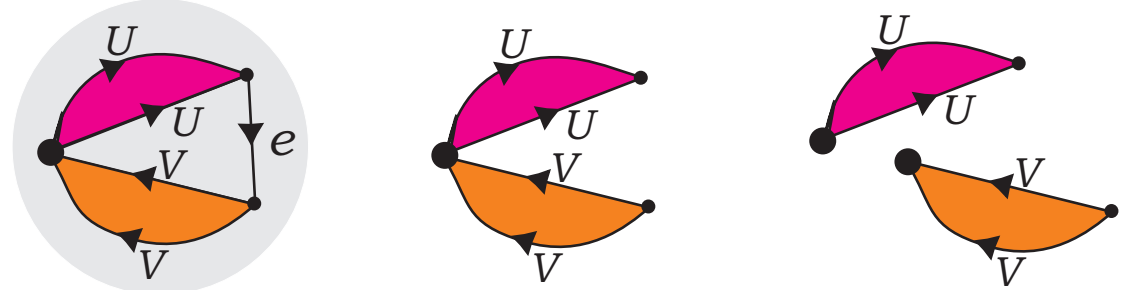

Figure 13. On the left is a sphere containing two 2 -cells with boundary $U e \mathrm{~V}$ meeting at the edge $e$. Note that one of these 2 -cells contains the 'back of the sphere' and cannot be fully seen. The middle diagram is obtained by removing the cancelable pair of 2 -cells and the 1 -cell $e$. On the right are the diagrams obtained by cutting along the large base vertex.

spherical diagram would show that $\mathrm{C} \rightarrow \mathrm{E}$ is not an immersion. Now we will use the diagrammatic reducibility to show that if $|\mathrm{C}| \geq 4$, then there exists a shorter immersed circle whose projection is nullhomotopic. The key point is that any cancelable pair must occur along an edge which occurs in the exact same position in the two based 2 -cells. For the remainder of the argument we shall suppose that the cyclic permutation of $\mathrm{W}$ is of the form $U e \mathrm{~V}$ where $e$ is the edge along which the cancelable pair meets.

We refer the reader to Figure 13 for the sequence of diagrams involved in the argument. We first remove the two open 2 -cells together with the open 1 -cell $e$. We then obtain a singular disc diagram whose boundary path is $U U^{-1} \mathrm{~V}^{-1} \mathrm{~V}$, and such that the original basepoint is at the initial point of both $U$ paths and the terminal point of both $\mathrm{V}$ paths. When we cut these two disc diagrams apart, and fold the $U U^{-1}$ and $\mathrm{V}^{-1} \mathrm{~V}$ paths together we obtain a pair of based spherical diagrams, which are obtained by folding together the boundary path of a cyclically ordered arrangement of 2 -cells around a basepoint.

Each of these diagrams corresponds to a proper subpath $\mathrm{P}^{\prime} \rightarrow \mathrm{E}$ of $\mathrm{C} \rightarrow$ $\mathrm{E}$ which is essential but whose projection $\mathrm{P}^{\prime} \rightarrow \mathrm{L}$ is nullhomotopic. There is therefore an immersed circle $\mathrm{C}^{\prime} \rightarrow \mathrm{E}$ such that $\left|\mathrm{C}^{\prime}\right| \leq\left|\mathrm{P}^{\prime}\right|<|\mathrm{C}|$, and such that the projection $\mathrm{C}^{\prime} \rightarrow \mathrm{E}$ is nullhomotopic. This contradicts the minimality of $|\mathrm{C}|$ and we have completed the proof that $\mathrm{E} \rightarrow \mathrm{L}$ is $\pi_{1}$-injective.

To see that $\overline{\mathrm{E}} \rightarrow \mathrm{L}$ and hence $\mathrm{E} \rightarrow \mathrm{L}$ is malnormal, observe that using the construction described above, for any closed immersed circle $\mathrm{C} \rightarrow \overline{\mathrm{E}}$, its projection $\mathrm{C} \rightarrow \hat{\mathrm{Z}}$ is the boundary path of a disc diagram $\mathrm{D}$ consisting of a cyclic arrangement of 2 -cells. If $\mathrm{E} \rightarrow \mathrm{L}$ is not malnormal, then there is a distinct pair of such immersed circles, and we can identify the boundaries of their corresponding diagrams to obtain a sphere $\mathrm{S}^{2} \rightarrow Y$ with no cancelable pairs.

$\mathbf{n}=3$ : For the case $n=3$, instead of choosing $p$ and $q$ to be antipodal vertices, we choose them so that $q=|\mathrm{W}| p$. We then choose $\mathrm{L}, \mathrm{R}$, and $\mathrm{E}$ in a manner analogous to the choice in the even case in Construction 3.1. Thus, L has 'length' $|\mathrm{W}|$ and $\mathrm{R}$ has 'length' $2|\mathrm{~W}|$. The map $\mathrm{E} \rightarrow \mathrm{R}$ satisfies $c(4)$ because it is exactly the same map as in the case $n=4$, therefore it is $\pi_{1}$-injective and 
malnormal. The map $\mathrm{E} \rightarrow \mathrm{L}$ is $\pi_{1}$-injective and malnormal because it is exactly the same map as in the case $n=2$.

Remark 4.2. I do not know whether the amalgamated subgroup in the 'antipodal' splitting is malnormal for the case $n=3$. However, one can show that it is a genuine splitting by showing that attaching maps satisfy $c(3)$. This follows from a result of Weinbaum which states that every positive word $\mathrm{W}$ can be cyclically permuted so that it is of the form $\mathrm{AB}$ where both $\mathrm{A}$ and $\mathrm{B}$ have the property that they appear in a unique way as a subword of the cyclic word $\mathrm{W}$. We refer the reader to [Wei90], and to [DH92] for a generalization to arbitrary words.

One might attempt to demonstrate that $\mathrm{E} \rightarrow \mathrm{L}$ satisfies $c(3)$ in this case by showing that the concatenation of three consecutive pieces is shorter than $\frac{3}{2}|\mathrm{~W}|$. However, this approach is doomed because of the example $\left\langle a, b \mid\left((a b)^{n} a\right)^{3}\right\rangle$ whose relator has the following three consecutive pieces: $(a b)^{n-1} \cdot a b a \cdot(a b)^{n-1}$.

\section{Positive one-relator groups that are not residually finite}

In this section we describe some examples which highlight the limitations of any general residual finiteness results for positive one-relator groups. We begin with an example due to Higman of a positive one-relator group which is not residually finite (see [Bau71]).

Example 5.1. The group $\langle t, b \mid b t b t t b t b t b\rangle$ is finitely generated but not Hopfian. It is therefore not residually finite by a result of Malćev (see [LS77]). Note that this presentation satisfies $\mathrm{C}^{\prime}\left(\frac{2}{5}\right)$ but does not satisfy $\mathrm{C}(4)$. The presentation $\langle a, t|$ $\left.t^{-1} a^{2} t a^{-3}\right\rangle$ discovered by Baumslag and Solitar [BS62], yields a group which is not Hopfian because the map induced by $a \mapsto a^{2}, t \mapsto t$ is surjective but not injective. Now, letting $b=t^{-1} a$ we obtain the presentation $\left\langle a, t, b \mid t^{-1} a^{2} t a^{3}, b=t^{-1} a\right\rangle$. And solving for $a=t b$ we obtain the presentation $\langle t, b \mid b t b t t b t b t b\rangle$ as above.

More generally, one can show that the positive one-relator group $\left\langle t, b \mid(b t)^{m}(t b)^{n}\right\rangle$ is not residually finite unless either $m=0, m=1, n=0, n=1$, or $m=n$.

We now describe $\mathrm{C}^{\prime}\left(\frac{1}{n}\right)$ positive one-relator groups which are not subgroup separable. Recall that a subgroup $H \subset \mathrm{G}$ is separable if it is the intersection of finite index subgroups of $G$. Note that $G$ is residually finite if and only if the trivial subgroup of $G$ is separable. The group $G$ is subgroup separable if every finitely generated subgroup $H \subset \mathrm{G}$ is separable.

A simple criterion for recognizing that a subgroup $H \subset \mathrm{G}$ is not separable is the existence of an element $t \in \mathrm{G}$ such that $t^{-1} H t$ is a proper subgroup of $H$ [BN74]. Indeed $H$ is then a proper subgroup of $t H t^{-1}$, but because a finite group cannot be conjugated properly into itself, these two subgroups of $G$ have the same image in any finite quotient.

We now provide for each $n$ a $\mathrm{C}^{\prime}\left(\frac{1}{n}\right)$ positive one-relator group which is not subgroup separable because it contains a finitely generated subgroup that is con- 
jugated properly into itself.

Example 5.2. Let $\mathrm{G}$ be the group given by the following presentation which is easily seen to satisfy the $\mathrm{C}^{\prime}\left(\frac{1}{n}\right)$ small cancellation condition:

$$
\left\langle a, t \mid a t t(a t)^{1} t a(a t)^{2} t a(a t)^{3} t a \cdots(a t)^{n-1} t a(a t)^{n-1} a\right\rangle
$$

We will show that the subgroup $H=\langle a t, t a\rangle$ is conjugated properly into itself by the element $t$ of $\mathrm{G}$. Consider the following two relations

$$
t^{-1}(a t)^{-1} t=(a t)^{1} t a(a t)^{2} \cdots t a(a t)^{n} \quad t^{-1}(t a) t=a t
$$

The first of these relations is readily seen to be equivalent to the relator in the presentation. The second relation is a free equivalence. These two relations show that $H$ is conjugated into itself by $t$, but to complete the proof, we must show that $H$ is conjugated properly into itself. By adding the generators $x=(a t)^{-1}$, and $y=t a$, we obtain the new presentation

$$
\left\langle a, t, x, y \mid t^{-1}(a t)^{-1} t=(a t)^{1} t a \cdots t a(a t)^{n}, x=(a t)^{-1}, y=t a\right\rangle .
$$

Substituting, we obtain the presentation

$$
\left\langle a, t, x, y \mid t^{-1} x t=x^{-1} y x^{-2} y \cdots y x^{-n}, x=(a t)^{-1}, y=t a\right\rangle .
$$

Now removing the generator $a=t^{-1} y$ we obtain the presentation

$$
\left\langle t, t, x, y \mid t^{-1} x t=x^{-1} y x^{-2} y x^{-3} \cdots y x^{-n}, t^{-1} y t=x^{-1}\right\rangle .
$$

Finally, note that when $n \geq 3$, the subgroup $\left\langle x, x^{-1} y x^{-2} y \cdots y x^{-n}\right\rangle$ is a proper subgroup of $\langle x, y\rangle$ and the above presentation is a properly ascending HNN extension. In particular, $H \cong\langle x, y\rangle$ is conjugated properly into itself by $t$, and is therefore not separable.

\section{Generically small-cancellation}

In this section we show that in a reasonable sense, positive one-relator groups are generically $\mathrm{C}^{\prime}(\alpha)$ for any $0<\alpha<1$. In particular, they are generically $\mathrm{C}^{\prime}\left(\frac{1}{6}\right)$. We note that finitely presented groups were shown to be generically wordhyperbolic by Ol'shanskiǐ [Ol'92].

Let $\mathrm{B}(r, n)$ be the set of one-relator presentations $\left\langle a_{1}, \ldots, a_{r} \mid \mathrm{R}\right\rangle$ where $\mathrm{R}$ is a positive word of length $n$. For simplicity, we will identify each such presentation with the cyclic word corresponding to its relator. We will not distinguish between relators which are cyclic permutations of each other, but we will distinguish between relators which differ by a permutation $\sigma$ of $\left\{a_{1}, \ldots, a_{r}\right\}$. So $\left\langle a_{1}, a_{2} \mid a_{1}^{2} a_{2}^{3}\right\rangle \not\left\langle a_{1}, a_{2} \mid a_{2}^{2} a_{1}^{3}\right\rangle$ but $\left\langle a_{1}, a_{2} \mid a_{1}^{2} a_{2}^{3}\right\rangle \sim\left\langle a_{1}, a_{2} \mid a_{1} a_{2}^{3} a_{1}\right\rangle$.

Let $Q(r, n) \subset \mathrm{B}(r, n)$ denote the subset consisting of presentations which fail to satisfy the $\mathrm{C}^{\prime}(\alpha)$ small-cancellation condition. Let $\mathcal{B}(r, n)$ denote $\cup_{m=1}^{n} \mathrm{~B}(r, m)$ and let $\mathcal{Q}(r, n)$ denote $\cup_{m=1}^{n} Q(r, m)$. And let $|\mathrm{B}(r, n)|,|Q(r, n)|,|\mathcal{B}(r, n)|$, and $|\mathcal{Q}(r, n)|$, denote the number of elements in these sets. 
Theorem 6.1. If $0<i<1$ then $\frac{|\mathcal{Q}(r, n)|}{|\mathcal{B}(r, n)|} \rightarrow 0$ as $n \rightarrow \infty$.

Proof. There are exactly $r^{n}$ positive words of length $n$ in the $r$ letters. The cyclic group $\mathbb{Z}_{n}$ acts on this set according to $a_{i_{1}} a_{i_{2}} \cdots a_{i_{n}} \mapsto a_{i_{2}} a_{i_{3}} \cdots a_{i_{n}} a_{i_{1}}$. Each orbit under this action has at most $n$ elements. Note that a positive word $\mathrm{W}^{k} \in \mathrm{B}(r, n)$ has exactly $|\mathrm{W}|$ elements in its orbit provided that $\mathrm{W}$ is not itself a proper power. It follows that $|\mathrm{B}(r, n)| \geq \frac{r^{n}}{n}$.

If $\mathrm{W} \in Q(r, n)$ then there is a piece $\mathrm{P}$ which appears as a subword of $\mathrm{W}$ in two different places and such that $|\mathrm{P}| \geq \alpha|\mathrm{W}|$. By possibly cyclically permuting $\mathrm{W}$, we may assume that $\mathrm{P}$ is an initial subword of $\mathrm{W}$.

In case there is no overlap between the two pieces then $\mathrm{W}=\mathrm{PSPT}$ where $\mathrm{S}$ and $\mathrm{T}$ are (possibly empty) positive words. It is clear that for each such positioning of $\mathrm{P}$ there are at most $r^{|\mathrm{P}|+|\mathrm{S}|+|\mathrm{T}|}$ possibilities, and since $|\mathrm{W}|=$ $|\mathrm{S}|+|\mathrm{T}|+2|\mathrm{P}|$ we see that there are at most $r^{|\mathrm{W}|-|\mathrm{P}|}$ possibilities.

In case there is overlap between the two occurrences of $\mathrm{P}$, then $\mathrm{W}=\mathrm{ABCD}$ where $\mathrm{AB}=\mathrm{P}=\mathrm{BC}$. Observe that $\mathrm{ABC}$ is completely determined by $\mathrm{A}$. So there are exactly $r^{|\mathrm{A}|} r^{|\mathrm{D}|}$ possibilities for each such positioning of $\mathrm{P}$. Since $\mathrm{BC}=\mathrm{P}$ we see that $|\mathrm{D}|=|\mathrm{W}|-|\mathrm{A}|-|\mathrm{P}|$, and so there are again, at most $r^{|\mathrm{A}|} r^{|\mathrm{D}|}=r^{|\mathrm{W}|-|\mathrm{P}|}$ possibilities.

Since there are at most $n=|\mathrm{W}|$ ways of positioning the second occurrence of $\mathrm{P}$, and because there are at most $r^{|\mathrm{W}|-|\mathrm{P}|}$ possibilities for each of these positions, we see that there are at most $n r^{|\mathrm{W}|-|\mathrm{P}|} \leq n r^{(1-\alpha) n}$ elements in $Q(r, n)$. Then

$$
\frac{|\mathcal{Q}(r, n)|}{|\mathcal{B}(r, n)|}=\frac{\sum_{m=1}^{n}|Q(r, m)|}{\sum_{m=1}^{n}|\mathrm{~B}(r, m)|} \leq \frac{\sum_{m=1}^{n} m r^{(1-\alpha) m}}{\sum_{m=1}^{n} \frac{r^{m}}{m}} \leq \frac{n^{2} r^{(1-\alpha) n}}{\frac{r^{n}}{n}}=\frac{n^{3}}{r^{\alpha n}}
$$

Note that the third inequality holds because the numerator increases whereas the denominator decreases. Finally, since $\frac{n^{3}}{r^{\alpha n}} \rightarrow \infty$ as $n \rightarrow \infty$, we are done.

\section{Acknowledgement}

I am grateful to the referee for many helpful comments that improved the exposition.

\section{References}

[Bau67] G. Baumslag, Residually finite one-relator groups, Bull. Amer. Math. Soc. 73 (1967), 618-620.

[Bau71] G. Baumslag, Positive one-relator groups, Trans. Amer. Math. Soc., 156 (1971), $165-183$.

[BN74] A. Blass, P. M. Neumann, An application of universal algebra in group theory, Michigan Math. J. 21 (1974), 167-169. 
[BP93] W. A. Bogley, S. J. Pride, Calculating generators of $\Pi_{2}$. In: C. Hog-Angeloni, W. Metzler, A. J. Sieradski, eds., Two-dimensional homotopy and combinatorial group theory, pages 157-188. Cambridge Univ. Press, Cambridge, 1993.

[BS62] G. Baumslag, D. Solitar, Some two-generator one-relator non-Hopfian groups, Bull. Amer. Math. Soc. 68 (1962), 199-201.

[DH92] A. J. Duncan, J. Howie, Weinbaum's conjecture on unique subwords of nonperiodic words, Proc. Amer. Math. Soc. 115(4) (1992), 947-954.

[Ego81] V. Egorov, The residual finiteness of certain one-relator groups. In: Algebraic systems, pages 100-121. Ivanov. Gos. Univ., Ivanovo 1981.

[GdlH90] Ghys, É., P. de la Harpe, (Eds.), Sur les groupes hyperboliques d'après Mikhael Gromov, Birkhäuser Boston Inc., Boston, MA, 1990. Papers from the Swiss Seminar on Hyperbolic Groups held in Bern, 1988.

[LS77] R. C. Lyndon, P. E. Schupp, Combinatorial group theory, Springer-Verlag, Berlin 1977. Ergebnisse der Mathematik und ihrer Grenzgebiete, Band 89

[MKS66] W. Magnus, A. Karrass, D. Solitar, Combinatorial group theory: Presentations of groups in terms of generators and relations. Interscience Publishers [John Wiley \& Sons, Inc.], New York-London-Sydney, 1966

[Ol'92] A. Yu. Ol'shanskiı̌, Almost every group is hyperbolic. Internat. J. Algebra Comput. 2(1) (1992), 1-17

[Pri83] S. J. Pride, Small cancellation conditions satisfied by one-relator groups, Math. Z. 184(2) (1983), 283-286.

[Sta83] J. R. Stallings, Topology of finite graphs, Invent. Math. 71 (1983), 551-565.

[Wei90] C. M. Weinbaum, Unique subwords in nonperiodic words, Proc. Amer. Math. Soc. $\mathbf{1 0 9}(3)$ (1990), 615-619.

[Wis] D. T. Wise, The residual finiteness of negatively curved polygons of finite groups. Preprint 1998, Submitted.

Daniel T. Wise

Dept. of Mathematics

Malott Hall

Cornell University

Ithaca, NY 14853

USA

e-mail: daniwise@math.cornell.edu

Current address:

Dept. of Mathematics and Statistics

Burnside Hall

McGill University

805 Sherbrooke Street

Montreal, Quebec, Canada

H3A2K6

(Received: August 4, 2000) 